\title{
Towards a seamlessly diagnosable expression for the energy flux associated with both equatorial and mid-latitude waves
}

\author{
Hidenori Aiki ${ }^{1,2^{*}}$, Richard J. Greatbatch ${ }^{3,4}$ and Martin Claus ${ }^{3}$
}

\begin{abstract}
For mid-latitude Rossby waves (RWs) in the atmosphere, the expression for the energy flux for use in a model diagnosis, and without relying on a Fourier analysis or a ray theory, has previously been derived using quasi-geostrophic equations and is singular at the equator. By investigating the analytical solution of both equatorial and mid-latitude waves, the authors derive an exact universal expression for the energy flux which is able to indicate the direction of the group velocity at all latitudes for linear shallow water waves. This is achieved by introducing a streamfunction as given by the inversion equation of Ertel's potential vorticity, a novel aspect for considering the energy flux. For ease of diagnosis from a model, an approximate version of the universal expression is explored and illustrated for a forced/dissipative equatorial basin mode simulated by a single-layer oceanic model that includes both mid-latitude RWs and equatorial waves. Equatorial Kelvin Waves (KWs) propagate eastward along the equator, are partially redirected poleward as coastal KWs at the eastern boundary of the basin, and then shed mid-latitude RWs that propagate westward into the basin interior. The connection of the equatorial and coastal waveguides has been successfully illustrated by the approximate expression of the group-velocity-based energy flux of the present study. This will allow for tropical-extratropical interactions in oceanic and atmospheric model outputs to be diagnosed in terms of an energy cycle in a future study.
\end{abstract}

Keywords: Group velocity, Model diagnosis, Equatorial Rossby waves, Equatorial mixed Rossby-gravity waves, Equatorial inertia-gravity waves, Equatorial Kelvin waves, Coastal Kelvin waves, Mid-latitude Rossby waves, Mid-latitude inertia-gravity waves, Tropical-extratropical interactions

\section{Introduction}

A feature of many phenomena in the equatorial oceans is the role played by equatorial Kelvin waves (KWs), examples being El Niño Southern Oscillation (ENSO; Philander 1989) and the so-called Atlantic Niño (Merle 1980). KWs propagate along the equator and are partially redirected into coastal KWs at the eastern boundary, where they can influence off-equatorial latitudes (e.g., Lübbecke et al. 2010) as well as excite extratropical Rossby waves (RWs) that subsequently propagate into the ocean interior (McPhaden and Ripa 1990; Isachsen et al. 2007).

*Correspondence: aiki@nagoya-u.jp

${ }^{1}$ Institute for Space-Earth Environmental Research, Nagoya University, Nagoya City, 464-8601 Aichi, Japan

${ }^{2}$ Application Laboratory, Japan Agency for Marine-Earth Science and Technology, Yokohama, Japan

Full list of author information is available at the end of the article
A striking example of this behavior is the equatorial basin mode (Cane and Moore 1981). For the gravest basin mode, the time scale is set by the time taken for an equatorial KW to propagate across the basin and for the reflected gravest long Rossby wave to return to the western boundary (that is $4 L / c$ where $L$ is the basin width and $c$ is the phase propagation speed for KWs). In addition to waves that are trapped on the equator, equatorial basin modes also feature coastal KWs that propagate along the eastern boundary and extratropical RWs that are excited by these KWs and refocus on the equator, as described by Schopf et al. (1981). There is growing evidence that equatorial basin modes play an important role in equatorial ocean dynamics. For example, basin modes have been associated with the equatorial deep jets (Johnson and Zhang 2003; Brandt et al. 2011; Claus et al. 2016) and with the semiannual (Thierry et al. 2004) and annual cycles (Brandt 
et al. 2016) in the equatorial Atlantic. However, the energy cycle associated with equatorial basin modes has received little attention and is an important factor when considering the forced/dissipative basin modes that one can relate to observations. A particularly interesting example is the upward energy propagation associated with the Atlantic equatorial deep jets (Johnson and Zhang 2003; Brandt et al. 2011; Mathiessen et al. 2015). Yet, the detailed energy cycle associated with the jets remains largely unknown.

One way to approach the energy flux is to use ray theory. However, ray theory is linked to the dispersion relation of a single type of wave and is not suitable for investigating the sequential connection of different types of waves that are associated with a basin mode. Likewise, a Fourier analysis is not suitable for the investigation of waves near the coastal boundaries of the ocean. In fact, it is only for mid-latitude inertia-gravity waves (IGWs) that the flux of wave energy has been diagnosed from oceanic model output (Cummins and Oey 1997; Niwa and Hibiya 2004; Furuichi et al. 2008). On the other hand, in the atmospheric literature, the model diagnosis of pseudomomentum (or wave activity) flux has been more popular than the model diagnosis of the energy flux (Hoskins et al. 1983; Plumb 1986; Takaya and Nakamura 1997; Nakamura and Solomon 2011).

Here, we seek a general expression that can be used to diagnose the energy flux associated with linear shallow water waves at all latitudes from model output. This manuscript is organized as follows. First we provide the theoretical background. Then, we present an analytical investigation that leads to a general expression for the energy flux that can indicate the exact profile of the group velocity times wave energy for both equatorial and mid-latitude waves. The utility of the universal expression of energy flux as a model diagnostic is illustrated for a forced/dissipative equatorial basin mode simulated by a single-layer model. The model diagnosis is achieved by introducing an inversion for the linearized version of Ertel's potential vorticity. This is a novel aspect for considering the energy flux in the presence of a coastal waveguide that connects the equatorial and mid-latitude regions.

\section{Theoretical background}

We use the shallow water equations for a single vertical normal mode (Gill 1982) appropriate to linear waves in a rotating frame of reference and in the absence of a mean flow. Let an arbitrary variable with an associated physical dimension be expressed by $A^{*}$, and let Cartesian-horizontal coordinates be labelled by the set of independent variables $x^{*}, y^{*}, t^{*}$, where each of $x^{*}, y^{*}$ increases eastward and northward, respectively, and $u^{*}, v^{*}$ are the corresponding horizontal components of velocity (a list of variables is given in Table 1$)^{1}$. The equations may then be written as

$$
\begin{aligned}
& \frac{\partial u^{*}}{\partial t^{*}}-f^{*} v^{*}+\frac{\partial p^{*}}{\partial x^{*}}=0, \\
& \frac{\partial v^{*}}{\partial t^{*}}+f^{*} u^{*}+\frac{\partial p^{*}}{\partial y^{*}}=0, \\
& \frac{\partial p^{*}}{\partial t^{*}}+c^{* 2}\left(\frac{\partial u^{*}}{\partial x^{*}}+\frac{\partial v^{*}}{\partial y^{*}}\right)=0,
\end{aligned}
$$

where $f^{*}=f_{0}^{*}+\beta^{*} y^{*}$ is the Coriolis parameter, $p^{*}=$ $p^{*}\left(x^{*}, y^{*}, t^{*}\right)$ corresponds to the pressure ${ }^{2}$ or geopotential, and $c^{*}$ is a uniform constant representing the propagation speed of nonrotating gravity waves for a given mode. Manipulation of (1a) - (1c) yields a prognostic equation for the linearized version of Ertel's potential vorticity (hereafter EPV and symbolized as $q^{*}$ ) to read

$$
\frac{\partial}{\partial t^{*}}(\underbrace{\frac{\partial v^{*}}{\partial x^{*}}-\frac{\partial u^{*}}{\partial y^{*}}-\frac{f^{*}}{c^{* 2}} p^{*}}_{\equiv q^{*}})+v^{*} \beta^{*}=0,
$$

which is applicable to waves at all latitudes, such as midlatitude RWs, mid-latitude IGWs, and equatorial waves [i.e., equatorial RWs and IGWs, equatorial Rossby-gravity waves (RGWs, i.e., Yanai waves), and equatorial KWs; Matsuno 1966; Yanai and Maruyama 1966], understanding $f_{0}^{*}=0$ for an equatorial $\beta$-plane and $\beta^{*}=0$ for a

\begin{tabular}{|c|c|}
\hline$f^{*}=f_{0}^{*}+\beta^{*} y^{*}$ & Coriolis parameter \\
\hline$c^{*}$ & Speed of long gravity wave \\
\hline$x, y, t$ & $\begin{array}{l}\text { Cartesian coordinates wherein } x \text { and } y \text { increase } \\
\text { eastward and northward }\end{array}$ \\
\hline$\langle\langle a, b\rangle\rangle$ & $\begin{array}{l}\text { Horizontal vector with eastward and northward } \\
\text { components } a \text { and } b\end{array}$ \\
\hline $\mathbf{v}=\langle\langle u, v\rangle\rangle$ & Horizontal velocity vector \\
\hline$\nabla \equiv\left\langle\left\langle\partial_{x}, \partial_{y}\right\rangle\right\rangle$ & Horizontal gradient operator \\
\hline$p$ & Pressure \\
\hline$q \equiv v_{x}-u_{y}-y p$ & $\begin{array}{l}\text { Linearized Ertel's potential vorticity: } q^{*} \equiv v_{x^{*}}^{*}-u_{y^{*}}^{*}- \\
\left(f^{*} / c^{* 2}\right) p^{*}\end{array}$ \\
\hline$\varphi$ & Solution of $\nabla^{2} \varphi-y^{2} \varphi-3 \varphi_{t t}=q$, see (16) \& (17a) \\
\hline$\varphi^{a p p}$ & Solution of $\nabla^{2} \varphi^{a p p}-y^{2} \varphi^{a p p}=q$, see (26a) \& (18a) \\
\hline$\left(u^{2}+v^{2}+p^{2}\right) / 2$ & Wave energy: $\left(u^{* 2}+v^{* 2}+p^{* 2} / c^{* 2}\right) / 2$ \\
\hline$\theta=k x-\omega t$ & Wave phase \\
\hline k & Zonal wavenumber \\
\hline$\omega$ & Wave frequency \\
\hline$H^{(n)}$ & Hermite polynomial, see endnote 1 \\
\hline$n$ & Meridional mode number of free equatorial waves \\
\hline $\bar{A}$ & Phase average of $A$ \\
\hline
\end{tabular}
mid-latitude $f$-plane. Both mid-latitude IGWs (i.e., $\beta^{*}=$

Table 1 List of symbols, where $A^{*}$ and $A$ are arbitrary quantities written dimensionally or non-dimensionally, respectively 
$0)$ and equatorial KWs (i.e., $v^{*}=0$ ) are characterized by $q^{*}=0$, as noted in Table 2 .

On the other hand, a prognostic equation for wave energy may be derived from (1a)-(1c) as

$$
\frac{\partial}{\partial t^{*}} \frac{1}{2}\left(\overline{u^{* 2}+v^{* 2}+\frac{p^{* 2}}{c^{* 2}}}\right)+\nabla^{*} \cdot\left\langle\left\langle\overline{u^{*} p^{*}}, \overline{v^{*} p^{*}}\right\rangle\right\rangle=0,
$$

where $\nabla^{*} \equiv\left\langle\left\langle\frac{\partial}{\partial x^{*}}, \frac{\partial}{\partial y^{*}}\right\rangle\right\rangle$ and the overbar symbol represents a phase-average operator (i.e., for a sinusoidal wave, $\overline{A^{*}}=$ 0 for $A^{*}=u^{*}, v^{*}$, and $p^{*}$ ) or a low-pass time filter (for this reason, we retain the local time derivative in (3) to allow for slow time variations in the general case).

For mid-latitude IGWs in the ocean and atmosphere, the group velocity vector points in the same direction as the energy flux vector in (3):

$$
\overline{\mathbf{V}^{*} p^{*}}=\left\langle\left\langle\overline{u^{*} p^{*}}, \overline{v^{*} p^{*}}\right\rangle\right\rangle,
$$

a property that has been exploited by Cummins and Oey (1997), Niwa and Hibiya (2004), and Furuichi et al. (2008) for a model diagnosis. However, for mid-latitude RWs, the vector in (4) does not point in the direction of the group velocity of the waves (Longuet-Higgins 1964; Masuda 1978; Cai and Huang 2013). In order to retrieve the correct direction for the energy flux associated with mid-latitude RWs, Orlanski and Sheldon (1993, hereafter OS93) have suggested to modify (3), without affecting the horizontal divergence of the energy flux, as

$$
\begin{aligned}
& \frac{\partial}{\partial t^{*}} \frac{1}{2}\left(\overline{u^{* 2}+v^{* 2}+\frac{p^{* 2}}{c^{* 2}}}\right)+ \\
& \nabla^{*} \cdot\left\langle\left\langle\overline{u^{*} p^{*}}+\frac{\partial}{\partial y^{*}}\left(\overline{\frac{p^{* 2}}{2 f^{*}}}\right), \overline{v^{*} p^{*}}-\frac{\partial}{\partial x^{*}}\left(\frac{\overline{p^{* 2}}}{2 f^{*}}\right)\right\rangle=0,\right.
\end{aligned}
$$

where each of $u^{*}$ and $v^{*}$ should be the sum of the geostrophic and ageostrophic components and $f^{*}=f_{0}^{*}+$

Table $\mathbf{2}$ Characteristics of different waves at various latitudes

\begin{tabular}{lll}
\hline & $\begin{array}{l}\overline{\mathbf{V}^{*} p^{*}} \text { parallel to } \\
\text { group velocity }\end{array}$ & $\begin{array}{l}q^{*}=0 \\
\left(\varphi^{\text {app* }}=0\right)\end{array}$ \\
\hline $\begin{array}{l}\text { Equatorial Rossby wave } \\
\begin{array}{l}\text { Equatorial mixed } \\
\text { Rossby-gravity wave }\end{array}\end{array}$ & No \\
$\begin{array}{l}\text { Equatorial inertia-gravity } \\
\text { wave }\end{array}$ & Roughly yes & No \\
$\begin{array}{l}\text { Equatorial Kelvin wave } \\
\text { Coastal Kelvin wave }\end{array}$ & Yes & Yes \\
$\begin{array}{l}\text { Mid-latitude Rossby wave } \\
\text { Mid-latitude inertia-gravity }\end{array}$ & No & Yes \\
wave & & No $\left(\varphi^{\text {app* }} \simeq p^{*} / f^{*}\right)$ \\
\hline
\end{tabular}

$\beta^{*} y^{*}$ is understood. The energy flux vector in (5) consists of two terms,

$$
\overline{\mathbf{V}^{*} p^{*}}-\nabla^{*} \times\left[\overline{p^{* 2}} /\left(2 f^{*}\right)\right] \mathbf{z},
$$

where $\overline{\mathbf{V}^{*} p^{*}}$ is as in the gravity wave literature (i.e., $\mathbf{V}^{*}$ is the sum of the geostrophic and ageostrophic components of velocity). The second term in (6) is the additional rotational component required to reproduce the direction of the group velocity of mid-latitude RWs ( $\mathbf{z}$ is the upward vertical unit vector). In Longuet-Higgins (1964), the second term of (6) has been expressed as $-\nabla^{*} \times\left[f^{*} \overline{\psi^{* 2}} / 2\right] \mathbf{z}$ where $\psi^{*}$ is a streamfunction based on the assumption of horizontally nondivergent velocity. This assumption is hardly used in modern oceanography owing to the smallness of the deformation radius. In quasi-geostrophic theory, $\psi^{*}=p^{*} / f^{*}$ from which the connection with (6) is clear.

The question naturally arises as to whether or not it is possible to find a general expression for the additional rotational flux, $\mathbf{R}^{*}$, that holds for waves at all latitudes and is such that the corresponding energy flux $\overline{\mathbf{V}^{*} p^{*}}+\mathbf{R}^{*}$ always points in the direction of the group velocity and thus constitutes a general expression for the energy flux associated with waves at all latitudes. This is the main subject of the present study. In this study, we focus on wave types for which the group velocity has been well formulated in the literature/textbook, as listed in Table 2. Of particular interest is the energy flux associated with equatorial RWs given that the expression in (6) is singular at the equator. The assumption of horizontally nondivergent velocity in Longuet-Higgins (1964) is also inappropriate for equatorial regions. In the next section, by investigating the analytical solution of equatorial waves, we derive an exact universal expression for the rotational flux which, after being added to $\overline{\mathbf{V}^{*} p^{*}}$, is able to indicate the direction of the group velocity for linear waves at all latitudes.

\section{Analytical investigation}

We begin by revisiting analytical expressions for the profile of the energy flux associated with equatorial waves. This investigation allows us to derive an expression for the energy flux that points in the direction of the group velocity for waves at all latitudes.

\section{Energy flux associated with equatorial waves}

We assume linear waves in the absence of a mean flow on an equatorial $\beta$-plane. As in Matsuno (1966) and Gill (1982), we use a time scale $1 / \sqrt{c^{*} \beta^{*}}$ and a length scale $\sqrt{c^{*} / \beta^{*}}$ to nondimensionalize the equation system (1a)(1c) to give

$$
\begin{aligned}
& u_{t}-y v+p_{x}=0, \\
& v_{t}+y u+p_{y}=0, \\
& p_{t}+u_{x}+v_{y}=0,
\end{aligned}
$$


where symbols without an asterisk indicate nondimensionalized quantities and subscripts indicate partial differentiations. Manipulation of (7a)-(7c) yields prognostic equations for EPV and wave energy in a nondimensionalized form to read,

$$
\begin{gathered}
\partial_{t}(\underbrace{v_{x}-u_{y}-y p}_{\equiv q})+v=0, \\
\left.\partial_{t}\left(\overline{u^{2}+v^{2}+p^{2}}\right) / 2+\nabla \cdot\langle\overline{u p}, \overline{v p}\rangle\right\rangle=0,
\end{gathered}
$$

where $\partial_{t} \equiv \frac{\partial}{\partial t}, \nabla \equiv\left\langle\left\langle\frac{\partial}{\partial x}, \frac{\partial}{\partial y}\right\rangle\right.$, and for $A=u, v$, or $p, \bar{A}=0$ for sinusoidally varying waves.

In what follows, we assume $v \not \equiv 0$ which is appropriate for equatorial RWs, RGWs, and IGWs (i.e., waves other than equatorial KWs). Then, we consider zonally propagating free waves with a relationship $v \propto \cos \theta, u \propto \sin \theta$, and $p \propto \sin \theta$ where $\theta \equiv k x-\omega t$ is wave phase with $k$ and $\omega$ being wavenumber and wave frequency, respectively. Substitution of these relationships to (7a)-(7c), followed by some manipulation, yields a characteristic equation for the meridional structure of $v$ to read,

$$
v_{y y}+\left(\omega^{2}-k^{2}-k / \omega-y^{2}\right) v=0 .
$$

Matsuno (1966) has derived a solution for $(7 a)-(7 c)$ and (10) to yield,

$$
\begin{aligned}
& v=\mathscr{A} \cos \theta \exp \left(-y^{2} / 2\right) H^{(n)}, \\
& u=\left(\omega y v_{\theta}-k v_{y \theta}\right) /\left(\omega^{2}-k^{2}\right), \\
& p=\left(k y v_{\theta}-\omega v_{y \theta}\right) /\left(\omega^{2}-k^{2}\right),
\end{aligned}
$$

where $\mathscr{A}$ is wave amplitude and the symbol $H^{(n)}$ is the Hermite polynomial with $n$ being the meridional mode number ${ }^{3}$. The subscript $\theta$ represents partial differentiation in terms of the wave phase [i.e., $v_{\theta} \equiv \partial v / \partial \theta=$ $\left.-\mathscr{A} \sin \theta \exp \left(-y^{2} / 2\right) H^{(n)}\right]$.

Substitution of (11a) to (10) yields,

$$
\omega^{3}-\left(k^{2}+2 n+1\right) \omega-k=0,
$$

which is a unified dispersion relation for equatorial RWs, RGWs, and IGWs. Partial differentiation of (12) with respect to wavenumber $k$ yields a unified expression for the group velocity of equatorial waves,

$$
\frac{\partial \omega}{\partial k}=\frac{2 k \omega+1}{3 \omega^{2}-\left(k^{2}+2 n+1\right)}=\frac{2 \omega^{2}+\omega / k}{2 \omega^{3} / k+1}
$$

where $2 \omega^{3} / k$ in the denominator has often been ignored in previous studies when focusing on low-frequency equatorial waves (e.g., equatorial RWs; Gill 1982).

We now investigate the energy flux associated with (7a)-(7c). It is known that, for zonally propagating equatorial waves, the meridional integral of $\overline{u p}$ is equal to the group velocity times the meridional integral of the wave energy (Philander 1989):

$$
\int_{-\infty}^{+\infty} \overline{u p} d y=(\partial \omega / \partial k) \int_{-\infty}^{+\infty}\left(\overline{u^{2}+v^{2}+p^{2}}\right) / 2 d y .
$$

It should be noted that the identity (14a) does not hold if it is evaluated without the meridional integral:

$$
\overline{u p} \neq(\partial \omega / \partial k)\left(\overline{u^{2}+v^{2}+p^{2}}\right) / 2 .
$$

For low-frequency equatorial waves (with $\omega<1-$ see Fig. 1-, i.e., all equatorial RWs and westward propagating RGWs), the meridional profiles of $\overline{u p}$ and $(\partial \omega / \partial k)\left(\overline{u^{2}+v^{2}+p^{2}}\right) / 2$ are shown by the dashed green and solid black lines, respectively, in Fig. 2. It is clear that, when compared at a given latitude, $\overline{u p}$ is not equal to the group velocity times wave energy. In particular, the meridional profile of $\overline{u p}$ is sign-indefinite for low-frequency equatorial waves (Fig. 2). On the other hand, as shown by the dashed green and solid black lines in Fig. 3 for highfrequency equatorial waves (with $\omega>1-$ see Fig. 1 -, i.e., all equatorial IGWs and eastward propagating RGWs), the meridional profile of $\overline{u p}$ provides a much better approximation for the group velocity times wave energy. The solid blue line, dashed orange line, and purple dots in Figs. 2 and 3 are explained later in the manuscript.

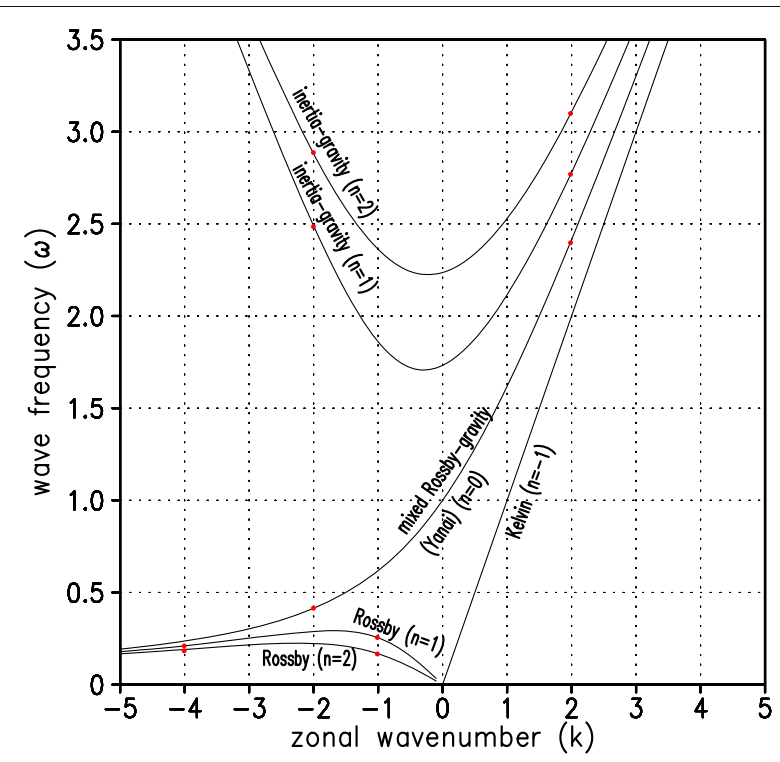

Fig. 1 Dispersion relation of free equatorial waves for a given meridional number $n$, as given by Eq. (12). Axes have been non-nondimensionalized by $\sqrt{\beta^{*} c^{*}}$ for wave frequency $\omega$ and by $\sqrt{\beta^{*} / c^{*}}$ for zonal wavenumber $k$. The red dots locate the parameters used to produce the 10 panels in Figs. 2 and 3 

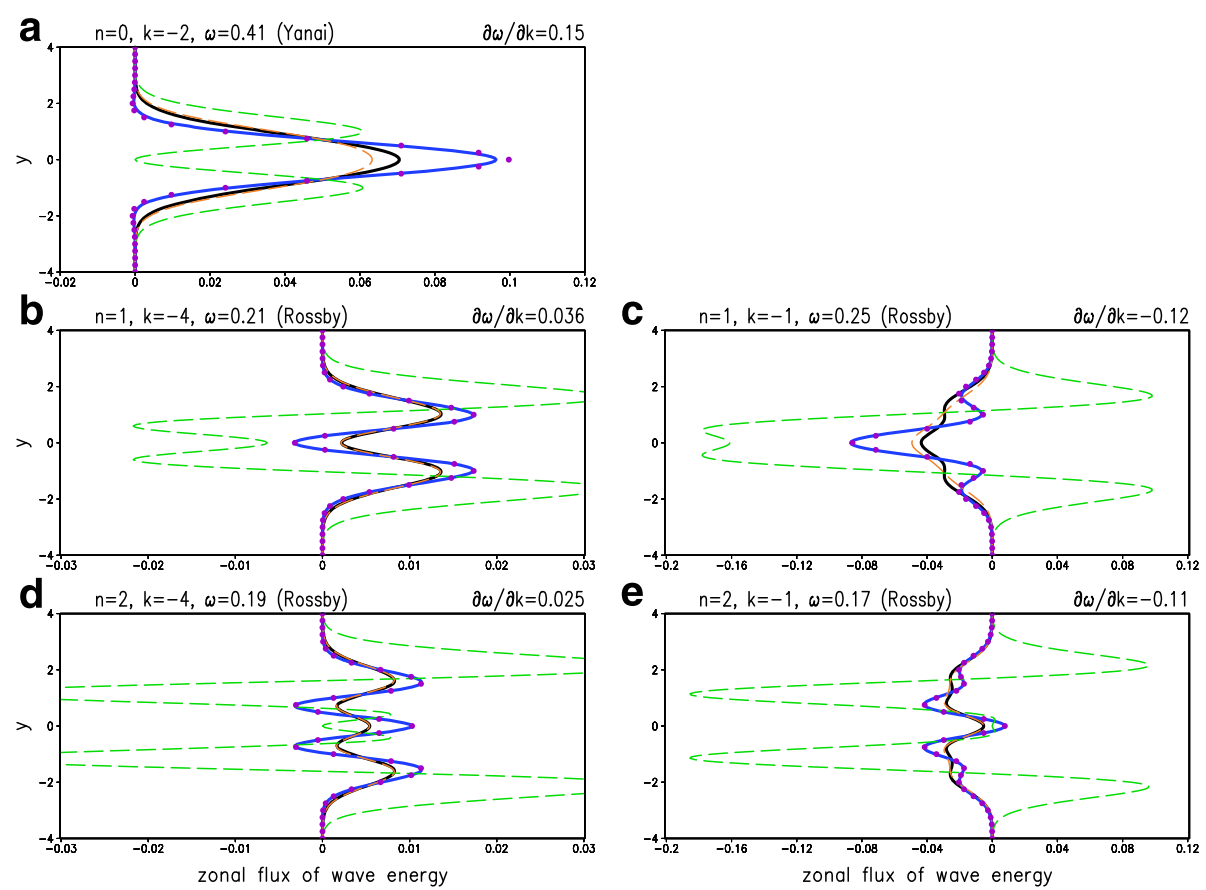

Fig. 2 Meridional profiles of the zonal component of phase-averaged energy flux associated with equatorial waves; the solid black line is $(\partial \omega / \partial k)\left(\overline{u^{2}+v^{2}+p^{2}}\right) / 2$, the dashed green line is $\overline{u p}$, the dashed orange line is $\overline{u p}+\left(\overline{p \varphi^{\mathrm{app}}} / 2+\overline{u_{t t} \varphi^{\mathrm{app}}}\right)_{y}$, the solid blue line is $\overline{u p}+\left(\overline{p \varphi^{\mathrm{app}}} / 2\right)_{y}$, and the purple dots are $(\omega / k)\left(\overline{E-v^{2}}\right)=(\omega / k)\left(\overline{u^{2}-v^{2}+p^{2}}\right) / 2$. All panels are for low-frequency equatorial waves with $\omega<1$ : a westward propagating RGWs, $\mathbf{b}$ short and $\mathbf{c}$ long RWs in the 1st meridional mode, and $\mathbf{d}$ short and $\mathbf{e}$ long RWs in the 2 nd meridional mode. The associated values of meridional-mode number $n$, zonal wavenumber $k$, wave frequency $\omega$, and group velocity $\partial \omega / \partial k$ are noted in each panel. For each of $\mathbf{a}-\mathbf{e}$, the wave amplitude $\mathscr{A}$ in (11a) has been set to normalize the meridional integral of wave energy: $\int_{-\infty}^{\infty}\left(\overline{u^{2}+v^{2}+p^{2}}\right) / 2 d y=1$
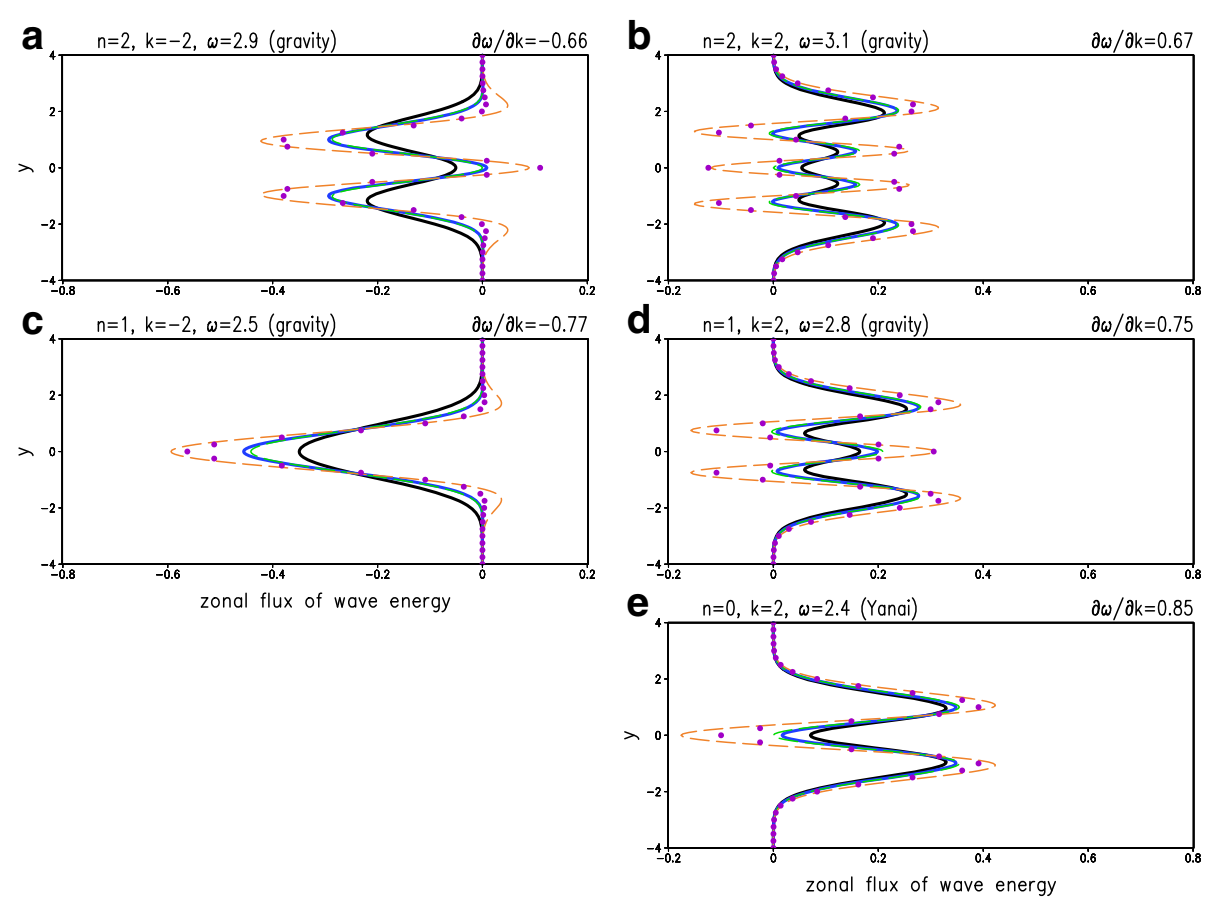

Fig. 3 Same as Fig. 2 except for high-frequency equatorial waves with $\omega>1$ : a westward and $\mathbf{b}$ eastward propagating IGWs in the 2nd meridional mode, $\mathbf{c}$ westward and $\mathbf{d}$ eastward propagating IGWs in the 1st meridional mode, and $\mathbf{e}$ eastward propagating RGWs 


\section{Identification of the additional rotational flux associated with equatorial waves}

It is useful to derive the analytical expression for the difference between the left and right hand sides of (14b). A first step for identifying the difference is to decompose the zonal component of $\overline{u p}$ into two parts, one that determines the meridional integral and one that does not affect it, as follows:

$$
\begin{aligned}
& \overline{u p} \\
& =\left[y^{2} \overline{v v}(\omega k)-y \overline{v_{y} v}\left(\omega^{2}+k^{2}\right)+\overline{v_{y} v_{y}}(\omega k)\right] /\left(\omega^{2}-k^{2}\right)^{2} \\
& =\left\{\overline{v_{y y} v}(\omega k)+\overline{v v}\left(\omega^{3} k-\omega k^{3}-k^{2}\right)\right. \\
& \left.-\left[(y \overline{v v} / 2)_{y}-\overline{v v} / 2\right]\left(\omega^{2}+k^{2}\right)+\overline{v_{y} v_{y}}(\omega k)\right\} /\left(\omega^{2}-k^{2}\right)^{2} \\
& =\left[\overline{v \nu}\left(2 \omega^{3} k-2 \omega k^{3}-k^{2}+\omega^{2}\right)+\left(\overline{v_{y} v}\right)_{y}(2 \omega k)\right. \\
& \left.-(y \overline{\nu v})_{y}\left(\omega^{2}+k^{2}\right)\right] /\left[2\left(\omega^{2}-k^{2}\right)^{2}\right] \\
& =\overline{v v}(2 \omega k+1) /\left[2\left(\omega^{2}-k^{2}\right)\right] \\
& +\left[\overline{v_{y} v}(2 \omega k)-y \overline{v v}\left(\omega^{2}+k^{2}\right)\right]_{y} /\left[2\left(\omega^{2}-k^{2}\right)^{2}\right],
\end{aligned}
$$

where the first equality has been derived using (11b)(11c) and $\overline{\sin \theta \sin \theta}=\overline{\cos \theta \cos \theta}$ and the second equality has been derived using (10). Note that it is the second of the two terms whose meridional integral is zero (noting that $v$ and $y v$ go to zero at large distances from the equator).

We now decompose the wave energy ${ }^{4}$ into two parts, one that determines the meridional integral and one does not. We then have

$$
\begin{aligned}
& \left(\overline{u^{2}+v^{2}+p^{2}}\right) / 2 \\
& =\overline{v v} / 2+\left[\left(y^{2} \overline{v v}+\overline{v_{y} \nu_{y}}\right)\left(\omega^{2}+k^{2}\right)\right. \\
& \left.-\left(y \overline{v_{y} v}\right)(4 k \omega)\right] /\left[2\left(\omega^{2}-k^{2}\right)^{2}\right] \\
& =\overline{v v} / 2+\left\{\left[y^{2} \overline{v v}-\overline{v_{y y} v}+\left(\overline{v_{y} v}\right)_{y}\right]\left(\omega^{2}+k^{2}\right)\right. \\
& \left.-(y \overline{\nu v})_{y}(2 k \omega)+(\overline{v v})(2 k \omega)\right\} /\left[2\left(\omega^{2}-k^{2}\right)^{2}\right] \\
& =\left[\overline{v \nu}\left(k^{4}-2 k^{2} \omega^{2}+\omega^{4}+\omega^{4}-k^{4}+k \omega-k^{3} / \omega\right)\right. \\
& \left.+\left(\overline{v_{y} v}\right)_{y}\left(\omega^{2}+k^{2}\right)-(y \overline{\nu v})_{y}(2 k \omega)\right] /\left[2\left(\omega^{2}-k^{2}\right)^{2}\right] \\
& =\overline{v v}\left(2 \omega^{2}+k / \omega\right) /\left[2\left(\omega^{2}-k^{2}\right)\right] \\
& +\left[\overline{v_{y} v}\left(\omega^{2}+k^{2}\right)-y \overline{\nu v}(2 k \omega)\right]_{y} /\left[2\left(\omega^{2}-k^{2}\right)^{2}\right] \text {, }
\end{aligned}
$$

where the first equality has been derived using (11b)(11c) and $\overline{\sin \theta \sin \theta}=\overline{\cos \theta \cos \theta}$, and the third equality has been derived using (10). As before, it is the second of the two terms whose meridional integral is zero. Using (14c)-(14d), we now obtain an analytical expression for the difference between the right and left hand sides of (14b) to yield

$$
\begin{aligned}
&(\partial \omega / \partial k)\left(\overline{u^{2}+v^{2}+p^{2}}\right) / 2-\overline{u p} \\
&=\frac{\left(\overline{v_{y} v}\right)_{y}}{2\left(\omega^{2}-k^{2}\right)^{2}}\left[\frac{\left(2 \omega^{2}+\omega / k\right)\left(\omega^{2}+k^{2}\right)}{2 \omega^{3} / k+1}-2 \omega k\right] \\
&-\frac{(y \overline{v v})_{y}}{2\left(\omega^{2}-k^{2}\right)^{2}}\left[\frac{\left(2 \omega^{2}+\omega / k\right) 2 k \omega}{2 \omega^{3} / k+1}-\left(\omega^{2}+k^{2}\right)\right] \\
&= \frac{\left(\overline{v_{y} v}\right)_{y}\left(2 \omega^{4}+2 \omega^{2} k^{2}+\omega^{3} / k+\omega k-4 \omega^{4}-2 \omega k\right)}{2\left(\omega^{2}-k^{2}\right)^{2}\left(2 \omega^{3} / k+1\right)} \\
&= \frac{(\overline{y \overline{\nu v}})_{y}\left(4 \omega^{3} k+2 \omega^{2}-2 \omega^{5} / k-2 \omega^{3} k-\omega^{2}-k^{2}\right)}{2\left(\omega^{2}-k^{2}\right)^{2}\left(2 \omega^{3} / k+1\right)} \\
&= \frac{\left[\overline{\left(\omega v_{y \theta}-k y v_{\theta}\right) v_{\theta}}\right]_{y}+\left[\bar{k}-2 \omega^{2}\right)-\left(y \overline{v_{\theta} v_{\theta}}\right)_{y}\left(1-2 \omega^{3} / k\right)}{2 k\left(\omega^{2}-k^{2}\right)\left(2 \omega^{3} / k+1\right)} \\
&= \frac{-\left(\overline{p v_{\theta}}\right)_{y}-\left(\overline{2 u_{t t} v_{\theta}}\right)_{y}}{2 k\left(1+2 \omega^{3} / k\right)},
\end{aligned}
$$

where the first and second equalities have been derived using (13), the third equality has been derived using $\overline{\cos \theta \cos \theta}=\overline{\sin \theta \sin \theta}$, and the last equality has been derived using (11b)-(11c). The last line of (14e) has been written as the meridional gradient of scalar quantities. Thus, the meridional integral of (14e) vanishes for equatorial waves (with a meridionally decaying structure) and is consistent with (14a).

Using (14e), we can now rewrite the zonal component of the group velocity times wave energy as

$$
\begin{aligned}
& (\partial \omega / \partial k)\left(\overline{u^{2}+v^{2}+p^{2}}\right) / 2=\overline{u p}+\left(\overline{p \varphi} / 2+\overline{u_{t t} \varphi}\right)_{y}, \\
& \varphi \equiv-v_{\theta} /\left(k+2 \omega^{3}\right),
\end{aligned}
$$

where the scalar quantity $\varphi$ has been introduced. We have confirmed that, as long as $\varphi$ is set by (15b), the meridional profile of the zonal energy flux, $\overline{u p}+\left(\overline{p \varphi} / 2+\overline{u_{t t} \varphi}\right)_{y}$, in $(15 \mathrm{a})$ is precisely identical to $(\partial \omega / \partial k)\left(\overline{u^{2}+v^{2}+p^{2}}\right) / 2$ for all types of equatorial waves in Figs. 2 and 3. Namely, all solid black lines in Figs. 2 and 3 may be drawn using either expression. As far as we know, (15a) and (15b) have not been mentioned in previous studies and therefore constitute a new result.

\section{Inversion equations for Ertel's potential vorticity}

The definition of $\varphi$, as given by (15b), is based on a Fourier expansion. However, we have found that (15b) may be rewritten into an expression which contains none of $\theta, k$, and $\omega$ to read

$$
\begin{aligned}
\nabla^{2} \varphi-y^{2} \varphi-3 \varphi_{t t} & =-v_{\theta} / \omega \\
& =q
\end{aligned}
$$


where $\nabla^{2} \equiv \partial_{x x}+\partial_{y y}$ is understood, the first line has been derived using (10), and the second line has been derived using (8) [i.e., $q_{t}=-\omega q_{\theta}=-v$ and thus $-\omega q_{\theta \theta}=$ $\omega q=-v_{\theta}$ ]. The new Eq. (16) of EPV is the cornerstone of the present study, because it suggests a possibility for the scalar quantity $\varphi$ to be estimated without using a Fourier analysis. This feature is important for identifying the direction of the energy flux of waves in the presence of coastal boundaries.

To summarize, in order to reproduce the profile of the group velocity times wave energy without relying on a Fourier analysis, we have obtained a new expression for the energy flux that has turned out to be associated with the streamfunction Eq. (16). Equation (16) may be rewritten into a dimensional form as

$$
\nabla^{* 2} \varphi^{*}-\left(f^{*} / c^{*}\right)^{2} \varphi^{*}-\left(3 / c^{* 2}\right) \varphi_{t^{*} t^{*}}^{*}=q^{*},
$$

where $\nabla^{*} \equiv\left\langle\left\langle\partial_{x^{*}}, \partial_{y^{*}}\right\rangle\right\rangle$ and $q^{*}=v_{x^{*}}^{*}-u_{y^{*}}^{*}-\left(f^{*} / c^{* 2}\right) p^{*}$. The exact profile of the group velocity times wave energy may be reproduced by the right hand side of (15a) and is here rewritten into a vector and dimensional form as

$$
\overline{\mathbf{V}^{*} p^{*}}-\nabla^{*} \times\left[\left(\overline{p^{*} \varphi^{*}}\right) / 2+\left(\overline{\left(\overline{u^{*} t^{*}} \varphi^{*}\right.}\right) / \beta^{*}\right] \mathbf{z} .
$$

The additional rotational flux in (17b) corrects the profile of the energy flux, without affecting the divergence of the energy flux. The quantity $\varphi^{*}$ in $(17 \mathrm{~b})$ is the solution of the accurate streamfunction Eq. (17a) associated with EPV in a dimensional form. We note in passing that for zonally propagating equatorial waves, as given by (11a)-(11c), $\overline{v^{*} p^{*}}$ vanishes owing to the phase relationship between $v^{*}$ and $p^{*}$ [see (11a) and (11c)] and the meridional component of the additional rotational flux, $-\left(\overline{p^{*} \varphi^{*}} / 2+\right.$ $\left.\overline{u_{t^{*}}^{*} \varphi^{*}} / \beta^{*}\right)_{x^{*}}$, also vanishes.

\section{Equatorial KWs}

So far, we have not investigated the energy flux of equatorial KWs. Since KWs are gravity waves, $\overline{\mathbf{V}}^{*} p^{*}$ becomes equal to the group velocity times wave energy. Namely, the additional rotational flux is absent. KWs are also characterized by $q^{*}=0$; hence, the EPV equation (17a) yields $\varphi^{*}=0$. The result is that, in the case of KWs, the expression for the energy flux, as given by $(17 \mathrm{~b})$ reduces to $\overline{\mathbf{V}^{*} p^{*}}$, which is consistent with the nature of gravity waves.

\section{Boundary conditions and the connection to mid-latitude regions}

Consider a basin with closed zonal boundaries (i.e., the eastern and western coastlines of a basin of arbitrary shape). It is clear that the flux $\overline{\mathbf{V}^{*} p^{*}}$ in (17b) has no component normal to the zonal boundaries. Hence, the additional rotational flux in (17b) should also have no component crossing the closed boundaries. This require- ment is satisfied in the present study by solving (17a) with a boundary condition of

$$
\varphi^{*}=0 .
$$

In a general situation in the ocean, waves propagating eastward along the equatorial waveguide are partially redirected poleward as KWs along the eastern boundary where they can shed RWs that then propagate westward into the ocean interior (Cane and Moore 1981; Philander 1989; Chelton and Schlax 1996; Isachsen et al. 2007).

We now investigate whether or not the set of (17a) and (17b) is applicable to off-equatorial regions where small-amplitude perturbations are characterized by either mid-latitude RWs or IGWs. For perturbations associated with mid-latitude RWs, the solution $\varphi^{*}$ of (17a) corresponds to the geostrophic streamfunction for which $\varphi^{*} \simeq$ $p^{*} / f^{*}$ is a reasonable approximation in an interior region (i.e., far from coastal boundaries), noting that $\nabla^{* 2} \varphi^{*}$ corresponds to $v_{x^{*}}^{*}-u_{\gamma^{*}}^{*}$. The result is that the energy flux in (17b) automatically reduces to the expression of OS93 for mid-latitude $\mathrm{RWs}^{5}$. On the other hand, if perturbations associated with mid-latitude IGWs are given, the inversion Eq. (17a) of EPV, which equals zero, yields, with $\varphi^{*}=0$ on the boundaries, $\varphi^{*}=0$ everywhere. Thus, the energy flux in (17b) automatically reduces to $\overline{\mathbf{V}^{*} p^{*}}$ which represents the group velocity of mid-latitude IGWs times wave energy. We conclude that the set of (17a) and (17b) can represent the exact profile of the group velocity times wave energy associated with both mid-latitude IGWs and RWs, which may be reconfirmed using almost the same procedure as in the "Identification of the additional rotational flux associated with equatorial waves" section. See Appendix 1 for details.

\section{Methods/Experimental}

The rest of this manuscript presents an example illustrating the diagnosis of the energy flux from a model. To be useful for our discussion, the exact universal expression for both equatorial and mid-latitude waves, as given by the set of (17a) and (17b), is hereafter referred to as the level-0 energy flux. In practice, the level-0 expression of the energy flux is not straightforward to compute from model output, since the second-order time derivative term in (17a) makes it difficult to solve for $\varphi^{*}$.

For the present study, we investigate the consequence of artificially removing the second-order time derivative term from (17a) to give

$$
\nabla^{* 2} \varphi^{\text {app } *}-\left(f^{*} / c^{*}\right)^{2} \varphi^{\text {app } *}=q^{*},
$$

which may be justified at least for low-frequency waves (e.g., both equatorial and mid-latitude RWs) based on scale analysis. The superscript of $\varphi^{\text {app* }}$ indicates that the solution of (18a) may be regarded as an approximation for 
the solution $\varphi^{*}$ of the accurate streamfunction Eq. (17a) associated with EPV. Then, we replace $\varphi^{*}$ in $(17 \mathrm{~b})$ with $\varphi^{\text {app* }}$ to read

$$
\overline{\mathbf{V}^{*} p^{*}}-\nabla^{*} \times\left[\left(\overline{p^{*} \varphi^{\mathrm{app} *}}\right) / 2+\left(\overline{u_{t^{*} t^{*}}^{*} \varphi^{\mathrm{app} *}}\right) / \beta^{*}\right] \mathbf{z},
$$

which is diagnosable ${ }^{6}$ from model output and is referred to as the level-1 expression of the energy flux in the present study. As shown by the dashed orange lines in Fig. 2, the level-1 expression provides a nice approximation for the group-velocity-based energy flux of lowfrequency equatorial waves, but not for high-frequency equatorial waves in Fig. 3. Next, with the form of the additional rotational flux $-\nabla^{*} \times\left[\overline{p^{* 2}} /\left(2 f^{*}\right)\right] \mathbf{z}$ in (6) in mind, we investigate the consequence of simplifying (18b) as

$$
\overline{\mathbf{V}^{*} p^{*}}-\nabla^{*} \times\left(\overline{p^{*} \varphi^{\mathrm{app} *}} / 2\right) \mathbf{z},
$$

which we refer to as the level-2 expression for the energy flux. As shown by the solid blue lines in Figs. 2 and 3, the level-2 expression provides an approximation for the group-velocity-based energy flux of both low- and highfrequency equatorial waves, although there can be some error. Further discussion of the level-2 approximation is given in Appendices 2 and 3 where it is noted that the level-2 approximation is comparable in accuracy to the pseudomomentum (or wave-activity) flux used in previous studies (Randel and Williamson 1990; Brunet and Haynes 1996; Fukutomi and Yasunari 2002; Wakata and Kitaya 2002; Kawatani et al. 2010).

We now contrast both the level-1 and level-2 energy fluxes with the expressions in previous studies, given by (6) and (4), using a solution from a linear shallow water model. This illustrates the potential of the expression given by (18b) and (18c) for use as a model diagnostic (see Table 3). Suitable for this purpose is an equatorial basin mode solution since it is associated with both equatorial and coastal waveguides as well as the radiation of mid-latitude RWs into the basin interior. Furthermore, as noted in the Introduction section, the equatorial basin mode, first studied by Cane and Moore (1981), has recently attracted attention because of its importance in the dynamics of the equatorial Atlantic Ocean. Indeed, the annual cycle, the semi-annual cycle, and the interannual variability associated with the Atlantic equatorial deep jets (Brandt et al. 2011) all appear to be resonant excitations of equatorial basin modes [see Brandt et al. (2016) and Claus et al. (2016) for more details].

\section{Model set-up}

To illustrate the importance of dissipation for explaining the observed cross-equatorial width of the equatorial deep jets, Greatbatch et al. (2012, hereafter G12) have simulated a forced/dissipative basin mode solution using a singlelayer reduced-gravity linear model. The model is set up in spherical coordinates, with a rectangular domain in latitude/longitude space of roughly the same width as the Atlantic Ocean at the equator (that is $55^{\circ}$ in longitude) and reaching to $10^{\circ} \mathrm{N} / \mathrm{S}$ on either side of the equator ${ }^{7}$. All lateral boundaries are closed. In both G12 and Claus et al. (2014, hereafter C14), the model has been forced by an idealized oscillatory forcing with a period of 4.5 years in the zonal momentum equation to mimic the forcing of the jets, together with a lateral mixing of momentum that provides dissipation. [See Ascani et al. (2015) for a discussion on the forcing of the equatorial deep jets, the details of which are not important here]. It should be noted that 4.5 years is roughly the time taken for an equatorial $\mathrm{KW}$ and the reflected long gravest equatorial RW, to travel across the basin for the vertical mode that is closest to resonance. As noted in G12 and C14, the (westward) propagation speed of equatorial long RWs is three times less than the (eastward) propagation speed of equatorial KWs [see the dispersion relation (12)].

Our model has been set up as in G12 and C14. The gravity wave speed is set equal to $c^{*}=0.17 \mathrm{~m} / \mathrm{s}$ [see the upper panel in Fig. 4 of C14]. The equatorial deformation radius becomes $\sqrt{c^{*} / \beta^{*}}=87 \mathrm{~km}$, with a consequence that disturbances further than a few degrees from the equator in our model experiment may be regarded as mid-latitude

\begin{tabular}{|c|c|c|c|c|}
\hline Approx. & Energy flux vector & Equation & Figs. $2 \& 3$ & Figs. $6 \& 7$ \\
\hline Level-0 & $\overline{\mathbf{V}^{*} p^{*}}-\nabla^{*} \times\left[\left(\overline{p^{*} \varphi^{*}}\right) / 2+\left(\overline{u_{t^{*} t^{*}}^{*} \varphi^{*}}\right) / \beta^{*}\right] \mathbf{z}$ & (15a), (17b), (23a) & Solid black & - \\
\hline Level-1 & $\overline{\mathbf{V}^{*} p^{*}}-\nabla^{*} \times\left[\left(\overline{p^{*} \varphi^{\text {app } *}}\right) / 2+\left(\overline{u_{t^{*} t^{*}}^{*} \varphi^{\text {app* }}}\right) / \beta^{*}\right] \mathbf{z}$ & $(18 b),(26 b)$ & Dashed orange & - \\
\hline Level-2 & $\overline{\mathbf{V}^{*} p^{*}}-\nabla^{*} \times\left(\overline{p^{*} \varphi^{\mathrm{app} *}}\right) / 2 \mathbf{z}$ & $(18 c),(26 c)$ & Solid blue & (c) \\
\hline QG & $\overline{\mathbf{V}^{*} p^{*}}-\nabla^{*} \times\left[\overline{p^{* 2}} /\left(2 f^{*}\right)\right] \mathbf{z}$ & $(5),(6)$ & - & (b) \\
\hline$f$-Plane & $\overline{\mathbf{V}^{*} p^{*}}$ & $(3),(4)$ & Dashed green & (a) \\
\hline
\end{tabular}

Table 3 List of energy flux vectors and EPV-based streamfunctions in dimensional form and their location in the text and figures

Definition of EPV-based streamfunctions

$\begin{array}{ll}\nabla^{* 2} \varphi^{*}-\left(f^{*} / c^{*}\right)^{2} \varphi^{*}-\left(3 / c^{* 2}\right) \varphi_{t^{*} t^{*}}^{*}=q^{*}, & \text { (15b), (16), (17a), (23c) } \\ \nabla^{* 2} \varphi^{\text {app } *}-\left(f^{*} / c^{*}\right)^{2} \varphi^{\text {app } *}=q^{*} & \text { (18a), (26a) }\end{array}$


Table 4 Parameters in the model experiment of the present study

\begin{tabular}{ll}
\hline Long gravity wave speed & $c^{*}=0.17 \mathrm{~m} / \mathrm{s}$ \\
Equatorial deformation radius & $\sqrt{c^{*} / \beta^{*}}=87 \mathrm{~km}$ \\
Equatorial inertial period & $2 \pi / \sqrt{c^{*} \beta^{*}}=37$ days \\
Forcing period & $T^{*}=4.5$ years \\
Forcing amplitude & $10^{-10} \mathrm{~m} / \mathrm{s}^{2}$ \\
Forcing area & Full domain \\
Domain size & $55^{\circ}$ (zonal) $\times 20^{\circ}$ (meridional) \\
Forcing Froude number & $(0.0023 \mathrm{~m} / \mathrm{s}) / c^{*}=0.014$ \\
Horizontal resolution & $0.1^{\circ}($ zonal $) \times 0.1^{\circ}$ (meridional) \\
Lateral eddy viscosity & $10 \mathrm{~m}^{2} / \mathrm{s}$ \\
\hline
\end{tabular}

RWs, even though they are part of the equatorial basin mode resonance. As in G12, our model has been formulated in a spherical coordinate system with a grid spacing of $0.1^{\circ}$ in both longitude and latitude. The coefficient ${ }^{8}$ of eddy viscosity has been set to $10 \mathrm{~m}^{2} / \mathrm{s}$. From an initial condition of no motion and no pressure anomaly, the model has been integrated for 20 cycles (i.e., 90 years) using the oscillatory forcing which is sufficient for a steady oscillatory state to be reached. Since the model code is fully non-linear, we have set the amplitude of the forcing to a small value, $1.0 \times 10^{-10} \mathrm{~m} / \mathrm{s}^{2}$ to ensure that linear dynamics prevails. Indeed, the magnitude of velocity associated with the gravest basin mode may be scaled as $10^{-10} \mathrm{~m} / \mathrm{s}^{2} \times 4.5$ years $/(2 \pi)=0.0023 \mathrm{~m} / \mathrm{s}$, which results in a Froude number of $(0.0023 \mathrm{~m} / \mathrm{s}) / \mathrm{c}^{*}=0.014$ (nondimensional). These parameters are summarized in Table 4. Below, we show results from an experiment which corresponds to the "full" case in G12. In particular, the oscillatory zonal forcing is spatially uniform and acts over the whole model domain. All the model results shown below are averages over the last model cycle.

\section{Results and discussion}

At each time step of the model output, we have calculated the EPV-based streamfunction $\varphi^{\text {app* } *}$ (contours in

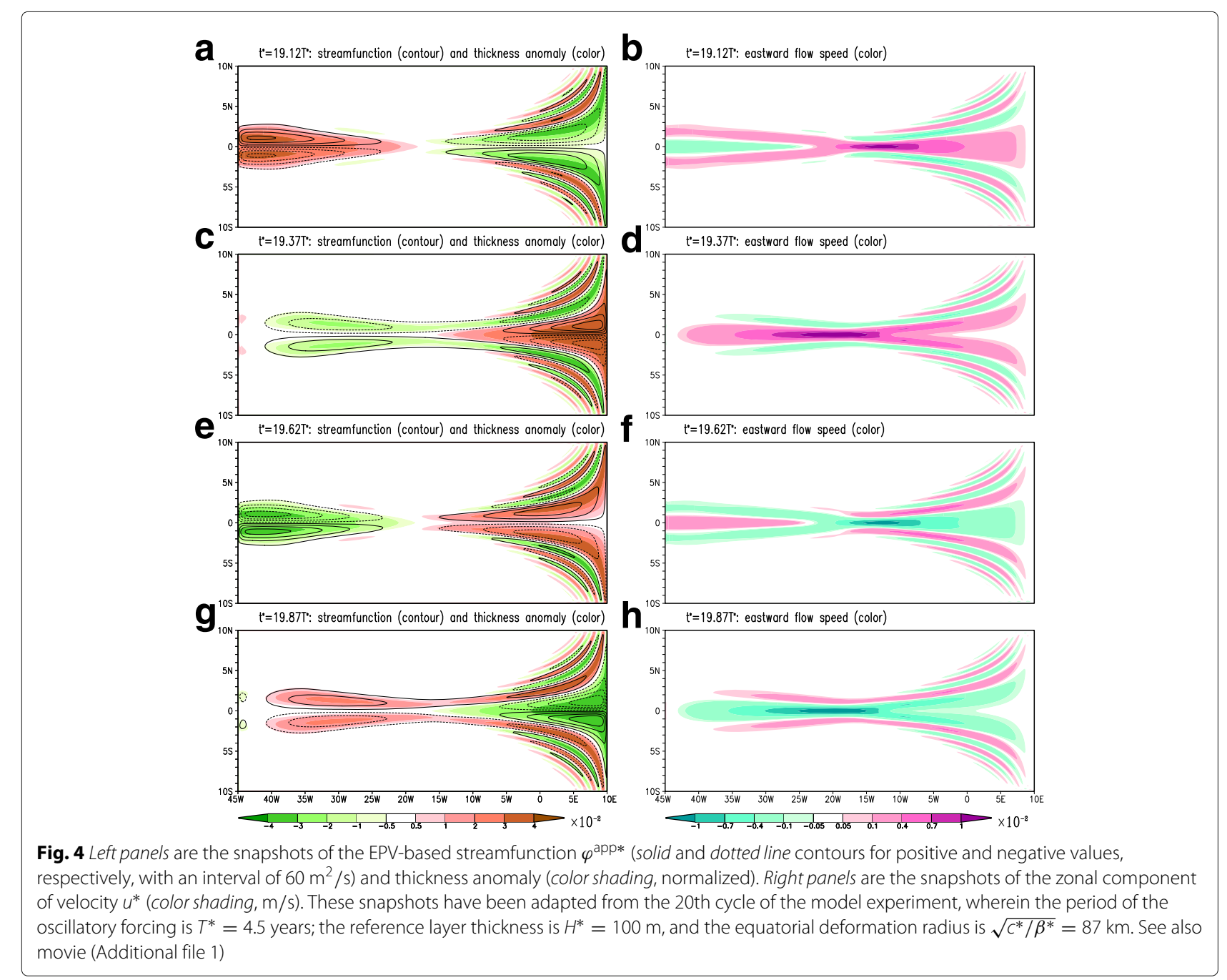


the left panels of Fig. 4) by solving the spherical coordinate version of (18a) with the boundary condition of $\varphi^{\text {app* }}=0$. The color shading in Fig. 4 shows the snapshots of thickness anomaly (left panels) and the zonal component of velocity $u^{*}$ (right panels). The movie of the model experiment is found in Additional file 1. RWs are identified by the correlation (anticorrelation) between the EPV-based streamfunction and thickness anomaly in the northern (southern) hemisphere. This follows from the correspondence between the EPV-based streamfunction and the geostrophic streamfunction for the case of midlatitude RWs, as noted earlier. As noted in G12 and C14, the (westward) propagation speed of equatorial long RWs is three times smaller than the (eastward) propagation speed of equatorial KWs [see the dispersion relation (12)]. It takes a three-quarter cycle (i.e., $3 T^{*} / 4$ ) for equatorial long RWs to travel westward from the eastern boundary to the western boundary of the model domain (see red lines in Fig. 5a). After reflection at the western boundary, it takes only a quarter of a cycle (i.e., $T^{*} / 4$ ) for equatorial KWs to travel eastward to the eastern boundary of the model domain (see blue lines in Fig. 5a), where some disturbances are deflected poleward along the eastern boundary to be the source of mid-latitude RWs which then propagate westward (Fig. 5b).

In Fig. 6, the divergence of the horizontal energy flux, given by $\nabla^{*} \cdot \overline{\mathbf{V}^{*} p^{*}}$, is shown for the whole model domain using color shading. Red indicates regions of a net energy input, and blue indicates regions of a net dissipation. It is clear that the main region of energy input is in the central part of the basin along the equator, where the strongest zonal velocities are found, and that the main regions of energy loss are associated with the RWs that radiate away from the eastern boundary. Arrows in Fig. 6a show the energy flux used in the gravity-wave literature, $\overline{\mathbf{V}^{*} p^{*}}$, which is mostly westward along the equator and eastward in the immediate off-equatorial region. This can be clearly seen in Fig. 7a which shows a blow-up of the eastern equatorial region. Figures $6 \mathrm{~b}$ and $7 \mathrm{~b}$ show the energy flux given by (6), which has been adapted from OS93, where only regions more than $1^{\circ}$ latitude away from the equator are plotted to avoid the singularity in the Coriolis parameter $f^{*}$ at the equator. From these figures (especially the blow-up of the eastern equatorial region in Fig. 7a, b), it is clear that the energy flux is strongly reversed when compared to $\overline{\mathbf{V}^{*} p^{*}}$ in the immediate off-equatorial region and is now strongly eastward in association with RWs that are radiated from the eastern boundary.

From Figs. $6 \mathrm{c}$ and $7 \mathrm{c}$, it is clear that when the set of Eqs. (18a), (18c) and (17c) is used to estimate the energy flux, the westward flux associated with the off-equatorial RWs is part of a recirculation of energy in the eastern part of the basin (Fig. 7c) with eastward energy flux along the equator and westward energy flux off the equator. The

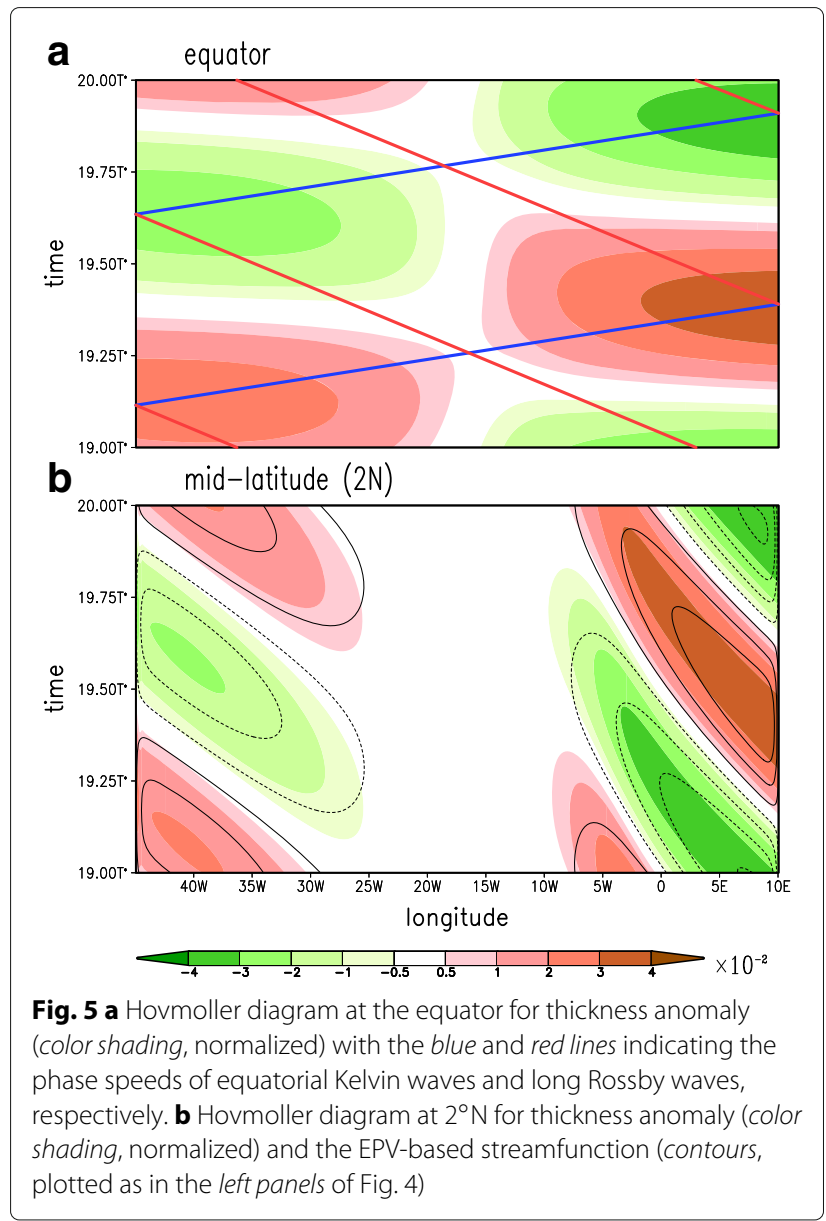

eastward flux along the equator in Figs. $6 \mathrm{c}$ and $7 \mathrm{c}$ is in the opposite direction to the westward $\overline{\mathbf{V}^{*} p^{*}}$ flux in Figs. 6a and $7 \mathrm{a}$ along the equator in the same region. This indicates the role of the rotational flux contribution in (18c) which counters the westward $\overline{\mathbf{V}^{*} p^{*}}$ flux along the equator. This westward flux is associated with the equatorial RWs but represents an overestimation of the energy flux associated with these waves (see Fig. 2). When the rotational flux is added, what emerges is the eastward flux associated with the KW which, in turn, leads to a poleward flux arising from KWs propagating along the eastern boundary and, in turn, leads to the westward flux associated with the off-equatorial RWs that are excited at the eastern boundary. Here, in terms of the transfer of wave energy, the equatorial waveguide has been connected to the eastern coastal waveguide and, in turn, to the basin interior at off-equatorial latitudes, which is at the heart of the present study.

Finally, we note that the forcing period of $T^{*}=4.5$ years is much longer than the equatorial inertial period of $2 \pi / \sqrt{c^{*} \beta^{*}}=37$ days. It can be said that the simulated equatorial basin mode consists of low-frequency equatorial waves, as in Fig. 2, and mid-latitude RWs. We recall the 


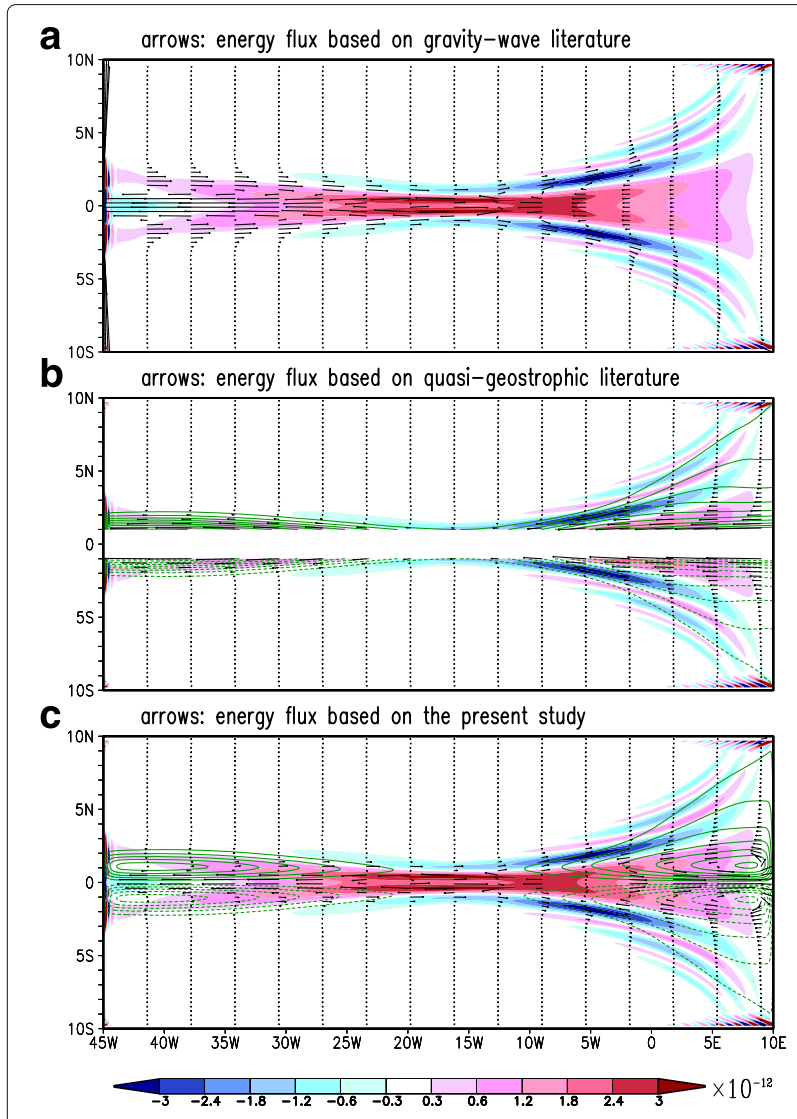

Fig. 6 Comparisons of three expressions for the horizontal flux of wave energy (arrows): a the pressure flux [see Eq. (3)], b the pressure flux plus the additional rotational flux of Orlanski and Sheldon (1993) [see Eq. (4)], and $\mathbf{c}$ the pressure flux plus the additional rotational flux of the present study [see Eq. (15)]. In both $\mathbf{b}$ and $\mathbf{c}$, the additional fluxes have been calculated using the rotation operator in the spherical coordinate system to be consistent with the model formulation. Green contours in $\mathbf{b}$ and $\mathbf{c}$ show the distributions of $\overline{p^{* 2}} /\left(2 f^{*}\right)$ and $\overline{p^{*} \varphi^{\text {app* }}} / 2$, respectively (solid and dotted lines indicate positive and negative values, respectively, with an interval of $10^{-2} \mathrm{~m}^{4} / \mathrm{s}^{3}$ ). Color shading in all panels shows the horizontal divergence of the time-averaged energy flux. Note that the additional rotational flux in $\mathbf{b}$ and $\mathbf{c}$ has no influence on the divergence. All quantities have been calculated from the output of the same experiment with a time-average between $t^{*}=19 T^{*}$ and $20 T^{*}$

small difference between the solid blue and dashed orange lines in Fig. 2, the former and the latter of which may be written as $\overline{u^{*} p^{*}}+\left(\overline{p^{*} \varphi^{\text {app } *}} / 2\right) y^{*}$ and $\overline{u^{*} p^{*}}+\left(\overline{p^{*} \varphi^{\text {app } *}} / 2+\right.$

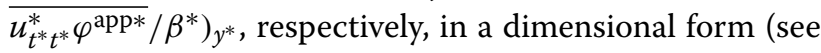
level-2 and level-1, respectively, in Table 3). Since arrows in Figs. $6 \mathrm{c}$ and $7 \mathrm{c}$ have been plotted using the expression which corresponds to the solid blue line in Fig. 2, we have checked for any improvement by using the expression which corresponds to the dashed orange lines in the same figure. The checking has been done by comparing the

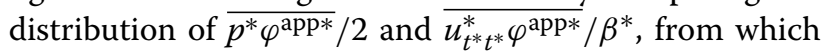

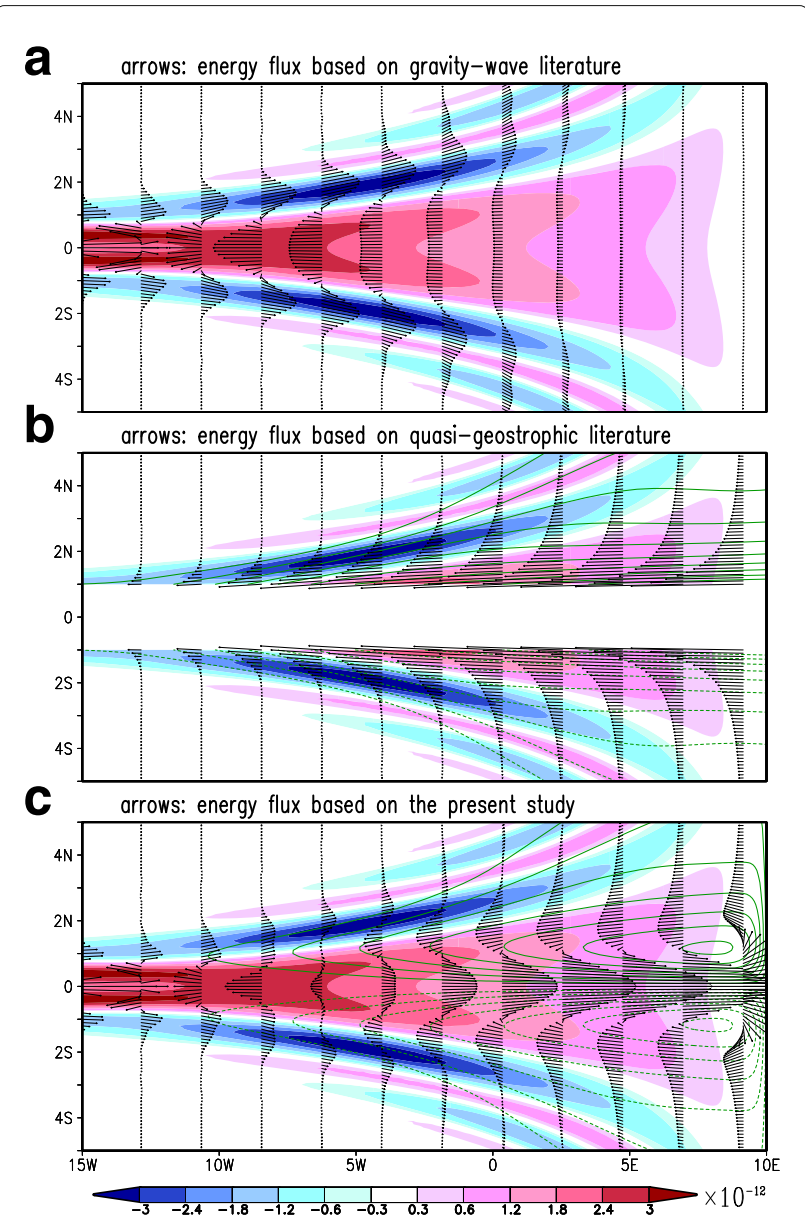

Fig. 7 Same as Fig. 6 except that this is an enlarged view of the eastern equatorial region of the model domain

we have learned that the latter quantity (not shown) is three orders of magnitude smaller than the former. Thus, we conclude that, in the diagnosis of the simulated basin mode, the expression of the energy flux, as given by (18c), has provided a nice approximation for the group velocity times wave energy.

\section{Conclusions}

In previous studies of the ocean, the energy flux of waves in model output has been diagnosed using $\overline{\mathbf{V}^{*} p^{*}}$, where $\mathbf{V}^{*}$ is the horizontal component of velocity perturbation and $p^{*}$ corresponds to the pressure perturbation. This is appropriate for understanding the energy flux associated with mid-latitude inertia-gravity waves (IGWs). For midlatitude Rossby waves (RWs), however, the direction of $\overline{\mathbf{V}^{*} p^{*}}$ differs from the group velocity and hence the energy flux, by a rotational vector flux with zero divergence. The rotational flux to be added to $\overline{\mathbf{V}^{*} p^{*}}$ for estimating the group velocity of mid-latitude RWs has previously been 
derived using quasi-geostrophic equations and is singular at the equator.

By investigating the analytical solution of both equatorial waves ("Analytical investigation" section) and midlatitude waves (Appendix 1), we have derived an exact universal $^{9}$ expression for the rotational flux which, after being added to $\overline{\mathbf{V}^{*} p^{*}}$, is able to indicate the profile of the group velocity times wave energy for linear waves at all latitudes. This is what we call the level-0 expression of the energy flux. The level-0 energy flux is written using the solution $\varphi^{*}$ of $(17 \mathrm{a})$, previously unmentioned in the literature, which we refer to as the accurate streamfunction associated with Ertel's potential vorticity (EPV). Equation (17a) is the cornerstone of the present study, because it suggests a possibility for the energy flux to be estimated (i) without using a Fourier analysis nor ray theory and (ii) in the presence of coastal boundaries, which will allow for tropical-extratropical interactions in model output to be diagnosed in terms of an energy cycle in a future study. Presently, the level-0 energy flux is not practical for use as a model diagnostic, since the secondorder time derivative term in (17a) makes it difficult to solve for $\varphi^{*}$. Thus, we hope that a future study is able to develop a numerical algorithm to solve (17a) for $\varphi^{*}$. We also note the need to extend the theory to a continuously stratified ocean and also to test out the theory in the presence of a sheared mean flow, both of which topics await a future study. This is a new step from the recent understanding of energetics in the atmosphere and ocean that had been focused on, for example, the global mapping of energy conversion rates associated with various physical processes (e.g., baroclinic and barotropic instabilities) and external forcing (Iwasaki 2001; Aiki and Richards 2008; Zhai et al. 2012).

The potential of our analysis as a model diagnostic is illustrated in the present study for a forced/dissipative equatorial basin mode simulated by a single-layer model. The model result includes both mid-latitude RWs (maintained by coastal KWs propagating poleward along the eastern boundary) and equatorial RWs (maintained by the reflection of equatorial KWs at the eastern boundary). We have used approximate expressions for the energy flux (what we call the level-1 and level- 2 energy fluxes) that is based on the inversion equation (18a) of EPV and which is shown to be good approximations to the level-0 expression in the case of the model run being considered. Since (18a) is seamlessly solvable at all latitudes with $\varphi^{\text {app* }}=0$ at coastlines, the source of the westward energy flux of mid-latitude RWs in the model output has been successfully illustrated in the present study. To our knowledge, this is the first attempt to diagnose the energy cycle of a tropical-extratropical interaction associated with the connection of the equatorial and coastal waveguides.

\section{Endnotes}

${ }^{1}$ While the energy flux of waves at all latitudes is considered in the present study, the pseudomomentum (or wave-activity) flux of waves at all latitudes is considered in Aiki et al. (2015, hereafter ATG15). Both the formulations of the present study and ATG15 may be reproduced even if a spherical coordinate system is used. The use of a Cartesian horizontal coordinate system in both the present study and ATG15 is for the purpose of simplicity, which will allow for the results of the two studies to be linked in a future study. A related discussion appears in Appendix 3.

${ }^{2}$ What we call pressure, energy, and momentum in the present study are actually dynamic pressure, energy density, and momentum density, respectively, following ATG15.

${ }^{3} d H^{(n)} / d y=2 n H^{(n-1)}, H^{(n+1)}=2 y H^{(n)}-2 n H^{(n-1)}$, $H^{(0)}=1, H^{(1)}=2 y, H^{(2)}=4 y^{2}-2, H^{(3)}=8 y^{3}-12 y$, $H^{(4)}=16 y^{4}-48 y^{2}+12$.

${ }^{4}$ The factor $\partial \omega / \partial k$ to calculate the energy flux is added in $(14 \mathrm{e})$.

${ }^{5}$ The second term in the square brackets of (17b) vanishes as $\overline{u_{t^{*} t^{*}}^{*} \varphi^{*}} \simeq \overline{\left(-p_{y^{*} t^{*} t^{*}}^{*} / f^{*}\right)\left(p^{*} / f^{*}\right)}=0$ where the phase relationship of plane waves is understood.

${ }^{6}$ We use the term "diagnosable" to indicate that the quantity is readily estimated from quantities in model output without relying on a Fourier analysis.

${ }^{7}$ In a related paper, Claus et al. (2014) also used this solution to investigate the influence of the barotropic mean flow on the Atlantic equatorial deep jets. The Atlantic equatorial deep jets are resonant with the gravest basin mode for a high-order baroclinic mode (typically the 15 th vertical normal mode) and consist of vertically stacked zonal jets that oscillate at a given depth with a period of around 4.5 years.

${ }^{8}$ This is lower than the value recommended by G12 for capturing the observed width of the deep jets but is chosen here since it is not so large as to prevent focusing of RWs on the equator. In the inviscid solution of Cane and Moore (1981), there is a singularity on the equator at the center of the basin due to RW focusing as described by Schopf et al. (1981).

${ }^{9}$ In the present manuscript, we have used the term "exact" to refer to the level-0 expression, in contrast to approximate expressions (i.e., level-1 and -2). Likewise, we have used the term "universal" to indicate the 
ability to handle all wave types in Table 2, for which the group velocity has been well formulated in the literature/textbook.

${ }^{10}$ Although it is not in the list of wave types in Table 2, IGWs on a mid-latitude $\beta$-plane may be characterized as $\alpha \ll 1, \delta^{2} \leq 1, \gamma^{2}<1$ where $\alpha \ll 1$ corresponds to (19b). Thus, the net content in the square brackets on the last line of (24c) becomes $O(1)$. Given $\alpha$ in front of $c^{*} \overline{v^{*} v^{*}}$ on the last line of (24c), we may justify (23d) for IGWs on a mid-latitude $\beta$-plane. It can be said that the right hand side of $(24 \mathrm{c})$ becomes significantly nonzero when the assumption of plane waves in the meridional direction becomes inconsistent (Anderson and Gill 1979).

${ }^{11}$ While the pseudomomentum flux itself $\left(\overline{E^{*}-v^{*} v^{*}}\right)$ is diagnosable from model output, the pseudomomentumflux-based expression of the energy flux $\left(\overline{E^{*}-v^{*} v^{*}}\right) \omega^{*} / k^{*}$ is not easily diagnosable from model output because of multiplication by the phase speed (see Appendix 3 for details).

\section{Appendix 1}

Is the streamfunction Eq. (17a) associated with EPV

\section{applicable to mid-latitude waves?}

Manipulation of the shallow water equation system (1a)(1c) yields a characteristic equation associated with the meridional component of velocity to read

$$
v_{t^{*} t^{*} t^{*}}^{*}-c^{* 2}\left(v_{x^{*} x^{*}}^{*}+v_{y^{*} y^{*}}^{*}\right)_{t^{*}}+f^{* 2} v_{t^{*}}^{*}-\beta^{*} c^{* 2} v_{x^{*}}^{*}=0,
$$

which is applicable to both mid-latitude and equatorial regions. In what follows, we consider plane waves on either an $f$-plane or a mid-latitude $\beta$-plane (i.e., $f^{*}=$ $f_{0}^{*}+\beta^{*} y^{*}$ and $\left.\left|f_{0}^{*}\right| \gg\left|\beta^{*} y^{*}\right|\right)$ and thus assume

$$
f^{* 2} \simeq f_{0}^{* 2}
$$

Then, (19a) may be simplified as

$$
v_{t^{*} t^{*} t^{*}}^{*}-c^{* 2}\left(v_{x^{*} x^{*}}^{*}+v_{y^{*} y^{*}}^{*}\right)_{t^{*}}+f_{0}^{* 2} v_{t^{*}}^{*}-\beta^{*} c^{* 2} v_{x^{*}}^{*}=0 .
$$

The Coriolis parameter $f_{0}^{*}$ in (19c) is constant that allows us to assume a horizontally monochromatic wave in a complex form

$$
v^{*}=\mathscr{A}^{*} e^{i \theta},
$$

where $i$ is the unit imaginary number, $\mathscr{A}^{*}$ is wave amplitude, and $\theta=k^{*} x^{*}+l^{*} y^{*}-\omega^{*} t^{*}$ is wave phase $\left(k^{*}\right.$ and $l^{*}$ are the zonal and meridional components of a wavenumber vector, respectively, and $\omega^{*}$ is wave phase). For simplicity, all $\mathscr{A}^{*}, k^{*}, l^{*}$, and $\omega^{*}$ are assumed to be constant. Substitution of (20a) to both (1a) and (1c) yields a solution for $u^{*}$ and $p^{*}$ to read

$$
\begin{aligned}
& u^{*}=\left(f^{*} \omega^{*} v_{\theta}^{*}+c^{* 2} k^{*} l^{*} v^{*}\right) /\left(\omega^{* 2}-c^{* 2} k^{* 2}\right), \\
& p^{*}=\left(f^{*} k^{*} v_{\theta}^{*}+\omega^{*} l^{*} v^{*}\right) c^{* 2} /\left(\omega^{* 2}-c^{* 2} k^{* 2}\right),
\end{aligned}
$$

where $f^{*}=f_{0}^{*}+\beta^{*} y^{*}$. On the other hand, substitution of (20a) to (19c) yields

$$
\omega^{* 3}-c^{* 2}\left(k^{* 2}+l^{* 2}\right) \omega^{*}-f_{0}^{* 2} \omega^{*}-\beta^{*} c^{* 2} k^{*}=0,
$$

which is a universal expression for the dispersion relation of the various types of waves in mid-latitude regions. For example, substitution of $\beta^{*}=0$ to (21) yields a classical dispersion relation for mid-latitude IGWs (i.e., waves on an $f$-plane), and substitution of $\omega^{* 2} \ll$ $c^{* 2} k^{* 2}$ to (21) yields a classical dispersion relation for mid-latitude RWs.

An expression for the zonal component of group velocity may be derived using (21) to read

$$
\begin{aligned}
\frac{\partial \omega^{*}}{\partial k^{*}} & =\frac{2 c^{* 2} k^{*} \omega^{*}+\beta^{*} c^{* 2}}{3 \omega^{* 2}-c^{* 2}\left(k^{* 2}+l^{* 2}\right)-f_{0}^{* 2}} \\
& =\frac{2 c^{* 2} \omega^{* 2} k^{*}+\beta^{*} c^{* 2} \omega^{*}}{2 \omega^{* 3}+\beta^{*} c^{* 2} k^{*}} .
\end{aligned}
$$

We now identify the content of $\left(\overline{A^{*} B^{*}}\right) y^{*}$ in the following equation:

$$
\overline{u^{*} p^{*}}+\left(\overline{A^{*} B^{*}}\right)_{y^{*}}=\frac{\partial \omega^{*}}{\partial k^{*}} \frac{1}{2}\left(\overline{u^{* 2}+v^{* 2}+\frac{p^{* 2}}{c^{* 2}}}\right),
$$

where each of $A^{*}$ and $B^{*}$ are quantities associated with the set of $u^{*}, v^{*}, p^{*}, c^{*}$, and $f^{*}$. A first step for investigating (22b) is to decompose $\overline{u^{*} p^{*}}$ into two parts: one that is associated with the numerator of (22a) and one that is written as the meridional derivative of a scalar quantity, as follows:

$$
\begin{aligned}
& \overline{u^{*} p^{*}}=\frac{\overline{v^{*} v^{*}}\left(f^{* 2}+c^{* 2} l^{* 2}\right) c^{* 2} \omega^{*} k^{*}}{\left(\omega^{* 2}-c^{* 2} k^{* 2}\right)^{2}} \\
& \simeq \frac{\overline{v^{*} v^{*}}\left(\omega^{* 3}-c^{* 2} k^{* 2} \omega^{*}-\beta^{*} c^{* 2} k^{*}\right) c^{* 2} k^{*}}{\left(\omega^{* 2}-c^{* 2} k^{* 2}\right)^{2}} \\
& =\frac{\overline{v^{*} v^{*}} c^{* 2} \omega^{*} k^{*}}{\left(\omega^{* 2}-c^{* 2} k^{* 2}\right)}-\frac{\left(f^{*} \overline{v^{*} v^{*}}\right)_{y^{*}} c^{* 4} k^{* 2}}{\left(\omega^{* 2}-c^{* 2} k^{* 2}\right)^{2}} \\
& =\frac{\overline{v^{*} v^{*}}\left(2 c^{* 2} \omega^{*} k^{*}+\beta^{*} c^{* 2}\right)}{2\left(\omega^{* 2}-c^{* 2} k^{* 2}\right)} \\
& -\frac{\left(f^{*} \overline{v^{*} v^{*}}\right)_{y^{*}} c^{* 2}\left(\omega^{* 2}+c^{* 2} k^{* 2}\right)}{2\left(\omega^{* 2}-c^{* 2} k^{* 2}\right)^{2}}
\end{aligned}
$$

where the first equality has been derived using both (20b)-(20c) and the set of $\overline{v^{*} v^{*}}=\overline{v_{\theta}^{*} v_{\theta}^{*}}$ and $\overline{v_{\theta}^{*} v^{*}}=0$ and the approximate equality in the middle has been derived using both the dispersion relation (21) and (19b). Then, we decompose the wave energy in (22b) into two parts, one that is associated with the denominator of (22a) and 
one that is written as the meridional derivative of a scalar quantity. We then have

$$
\begin{aligned}
& \frac{1}{2}\left(\overline{\left.u^{* 2}+v^{* 2}+\frac{p^{* 2}}{c^{* 2}}\right)}\right. \\
&=\frac{\overline{v^{*} v^{*}}\left(\omega^{* 2}-c^{* 2} k^{* 2}\right)^{2}}{2\left(\omega^{* 2}-c^{* 2} k^{* 2}\right)^{2}} \\
&+\frac{\overline{v^{*} v^{*}}\left(\omega^{* 2} f^{* 2}+c^{* 4} k^{* 2} l^{* 2}+k^{* 2} f^{* 2} c^{* 2}+\omega^{* 2} l^{* 2} c^{* 2}\right)}{2\left(\omega^{* 2}-c^{* 2} k^{* 2}\right)^{2}} \\
&= \frac{\overline{v^{*} v^{*}}\left[\left(\omega^{* 2}-c^{* 2} k^{* 2}\right)^{2}+\left(f^{* 2}+c^{* 2} l^{* 2}\right)\left(\omega^{* 2}+c^{* 2} k^{* 2}\right)\right]}{2\left(\omega^{* 2}-c^{* 2} k^{* 2}\right)^{2}} \\
& \simeq \frac{\overline{v^{*} v^{*}}\left(\omega^{* 2}-c^{* 2} k^{* 2}\right)^{2}}{2\left(\omega^{* 2}-c^{* 2} k^{* 2}\right)^{2}} \\
&+\frac{\overline{v^{*} v^{*}}\left(\omega^{* 2}-c^{* 2} k^{* 2}-\beta^{*} c^{* 2} k^{*} / \omega^{*}\right)\left(\omega^{* 2}+c^{* 2} k^{* 2}\right)}{2\left(\omega^{* 2}-c^{* 2} k^{* 2}\right)^{2}} \\
&= \frac{\left(f^{*} v^{*} v^{*}\right)_{y^{*}} c^{* 2} k^{*}\left(\omega^{* 2}+c^{* 2} k^{* 2}\right)}{\left(\omega^{* 2}-c^{* 2} k^{* 2}\right)}-\frac{2 \omega^{*}\left(\omega^{* 2}-c^{* 2} k^{* 2}\right)^{2}}{2\left(\omega^{* 2}-c^{* 2} k^{* 2}\right)^{2}}, \\
&= \frac{\left(f^{*} v^{*}\left(2 \omega^{* 3}+\beta^{*} c^{* 2} k^{*}\right)\right.}{2 \omega^{*}\left(\omega^{* 2}-c^{* 2} k^{* 2}\right)}-\frac{c^{* 2} \omega^{*} k^{*}}{2\left(\omega^{*}\right.}
\end{aligned}
$$

where the first equality has been derived using both (20b)-(20c) and the set of $\overline{v^{*} v^{*}}=\overline{v_{\theta}^{*} v_{\theta}^{*}}$ and $\overline{v_{\theta}^{*} v^{*}}=0$ and the approximated equality in the middle has been derived using both the dispersion relation (21) and (19b). The set of (22c) and (22d) allows us to identify the content of $\left(\overline{A^{*} B^{*}}\right)_{y^{*}}$ in $(22 \mathrm{~b})$ to read

$$
\begin{aligned}
& \frac{\partial \omega^{*}}{\partial k^{*}} \frac{1}{2}\left(\overline{u^{* 2}+v^{* 2}+\frac{p^{* 2}}{c^{* 2}}}\right)-\overline{u^{*} p^{*}} \\
& \simeq \frac{-\left(f^{*} \overline{\nu^{*} v^{*}}\right)_{y^{*} c^{* 2}}}{2\left(\omega^{* 2}-c^{* 2} k^{* 2}\right)^{2}}\left\{\frac{\left(2 c^{* 2} \omega^{* 2} k^{*}+\beta^{*} c^{* 2} \omega\right) 2 k^{*} \omega^{*}}{2 \omega^{* 3}+\beta^{*} c^{* 2} k^{*}}\right. \\
& \left.-\left(\omega^{* 2}+c^{* 2} k^{* 2}\right)\right\} \\
& =\frac{-\left(f^{*} \overline{v^{*} v^{*}}\right)_{y^{*}} c^{* 2}}{2\left(\omega^{* 2}-c^{* 2} k^{* 2}\right)^{2}}\left\{\frac{\left(4 c^{* 2} \omega^{* 3} k^{* 2}+2 \beta^{*} c^{* 2} \omega^{* 2} k^{*}\right)}{2 \omega^{* 3}+\beta^{*} c^{* 2} k^{*}}\right. \\
& \left.+\frac{\left(-2 \omega^{* 5}-2 c^{* 2} \omega^{* 3} k^{* 2}-\beta^{*} c^{* 2} \omega^{* 2} k^{*}-\beta^{*} c^{* 4} k^{* 3}\right)}{2 \omega^{* 3}+\beta^{*} c^{* 2} k^{*}}\right\} \\
& =\frac{-\left(f^{*} \overline{v_{\theta}^{*} v_{\theta}^{*}}\right)_{y^{*}} c^{* 2}\left(\beta^{*} c^{* 2} k-2 \omega^{* 3}\right)}{2\left(\omega^{* 2}-c^{* 2} k^{* 2}\right)\left(2 \omega^{* 3}+\beta^{*} c^{* 2} k^{*}\right)} \\
& =\frac{-\left(f^{*} \overline{v_{\theta}^{*} v_{\theta}^{*}}\right)_{y^{*}} c^{* 2}\left[1-2 \omega^{* 3} /\left(\beta^{*} c^{* 2} k^{*}\right)\right]}{2\left(\omega^{* 2}-c^{* 2} k^{* 2}\right)\left[2 \omega^{* 3} /\left(\beta^{*} c^{* 2} k^{*}\right)+1\right]} \\
& =\frac{-\left[\overline{\left(f^{*} k^{*} v_{\theta}^{*}+\omega^{*} l^{*} v^{*}\right) c^{* 2} v_{\theta}^{*}}\right]_{y^{*}}}{2 k^{*}\left(\omega^{* 2}-c^{* 2} k^{* 2}\right)\left[2 \omega^{* 3} /\left(\beta^{*} c^{* 2} k^{*}\right)+1\right]} \\
& +\frac{\left[\overline{\left(f^{*} \omega^{*} v_{\theta}^{*}+c^{* 2} k^{*} l^{*} v^{*}\right) v_{\theta}^{*}}\right]_{y^{*}} 2 \omega^{* 2} / \beta^{*}}{2 k^{*}\left(\omega^{* 2}-c^{* 2} k^{* 2}\right)\left[2 \omega^{* 3} /\left(\beta^{*} c^{* 2} k^{*}\right)+1\right]} \\
& =\frac{-\left(\overline{p^{*} v_{\theta}^{*}}\right)_{y^{*}}-\left(\overline{2 u_{t^{*}}^{*} v_{\theta}^{*}}\right)_{y^{*}} / \beta^{*}}{2 k^{*}\left[1+2 \omega^{* 3} /\left(\beta^{*} c^{* 2} k^{*}\right)\right]},
\end{aligned}
$$

where the last equality has been derived using (20a)-(20c). Equation (22e) may be rewritten as

$$
\begin{aligned}
\overline{u^{*} p^{*}}+ & \left(\overline{p^{*} \varphi^{*}} / 2+\overline{u_{t^{*} t^{*}}^{*} \varphi^{*}} / \beta^{*}\right)_{y^{*}} \\
& =\frac{\partial \omega^{*}}{\partial k^{*}} \frac{1}{2}\left(\overline{u^{* 2}+v^{* 2}+\frac{p^{* 2}}{c^{* 2}}}\right),
\end{aligned}
$$

where

$$
\varphi^{*} \equiv \frac{-v_{\theta}^{*}}{k^{*}+2 \omega^{* 3} /\left(\beta^{*} c^{* 2}\right)},
$$

has been introduced. The definition of $\varphi^{*}$, as given by (23b), is based on a Fourier expansion and may be rewritten into an expression which contains none of $\theta, k^{*}, l^{*}$, and $\omega^{*}$ to read

$$
\begin{aligned}
\nabla^{* 2} \varphi^{*}-\left(f_{0}^{*} / c^{*}\right)^{2} \varphi^{*}-\left(3 / c^{* 2}\right) \varphi_{t^{*} t^{*}}^{*} & =-\beta^{*} v_{\theta}^{*} / \omega^{*} \\
& =q^{*}
\end{aligned}
$$

where the first equality has been derived using (20b) and the second equality has been derived using (2) [i.e., $q_{t^{*}}^{*}=$ $-\omega^{*} q_{\theta}^{*}=-\beta^{*} v^{*}$ and thus $\left.-\omega^{*} q_{\theta \theta}^{*}=\omega^{*} q^{*}=-\beta^{*} v_{\theta}^{*}\right]$. As far as we know, the set of (23a) and (23c) has not been mentioned in previous studies for mid-latitude waves and has turned out to be almost the same as the set of (17b) and (17a) that has been derived for equatorial waves.

We now consider the meridional flux of wave energy. We would like to show that

$$
\begin{aligned}
\overline{v^{*} p^{*}}-\underbrace{\left(\overline{p^{*} \varphi^{*}} / 2+\overline{u_{t^{*} t^{*}}^{*} \varphi^{*}} / \beta^{*}\right)_{x^{*}}}_{0} \\
=\frac{\partial \omega^{*}}{\partial l^{*}} \frac{1}{2}\left(\overline{u^{* 2}+v^{* 2}+\frac{p^{* 2}}{c^{* 2}}}\right) .
\end{aligned}
$$

It turns out that the second term on the left hand side, associated with the additional rotational flux, vanishes when evaluated using the analytical solution of waves [i.e., $\left(\overline{v^{*} v^{*}}\right)_{x^{*}}=k \overline{v_{\theta}^{*} v^{*}}=0$ ], which is as in Longuet-Higgins (1964). This is attributed to the assumption of all $\mathscr{A}^{*}$, $k^{*}, l^{*}$, and $\omega^{*}$ being constant in particular in the zonal direction. An expression for the meridional component of group velocity may be derived from (21) to read

$$
\begin{aligned}
\frac{\partial \omega^{*}}{\partial l^{*}} & =\frac{2 c^{* 2} l^{*} \omega^{*}}{3 \omega^{* 2}-c^{* 2}\left(k^{* 2}+l^{* 2}\right)-f_{0}^{* 2}} \\
& =\frac{2 c^{* 2} l^{*} \omega^{* 2}}{2 \omega^{* 3}+\beta^{*} c^{* 2} k^{*}} .
\end{aligned}
$$

Then, we calculate the left hand side of (23d) using (20a)(20b) as

$$
\overline{v^{*} p^{*}}=\frac{\overline{v^{*} v^{*}} c^{* 2} \omega^{*} l^{*}}{\omega^{* 2}-c^{* 2} k^{* 2}},
$$

where $\overline{v_{\theta}^{*} v^{*}}=0$ has been used. We now calculate the difference of the meridional component of the group velocity 
times wave energy and $\overline{v^{*} p^{*}}$ using the set of (22d), (24a), and (24b) to yield

$$
\begin{aligned}
& \frac{\partial \omega^{*}}{\partial l^{*}} \frac{1}{2}\left(\overline{u^{* 2}+v^{* 2}+\frac{p^{* 2}}{c^{* 2}}}\right)-\overline{v^{*} p^{*}} \\
& =-\frac{2 c^{* 4} k^{*} l^{*} \omega^{* 3}}{\left(2 \omega^{* 3}+\beta^{*} c^{* 2} k^{*}\right)} \frac{\left(f \overline{v^{*} v^{*}}\right)_{y^{*}}}{\left(\omega^{* 2}-c^{* 2} k^{* 2}\right)^{2}} \\
& =-\frac{2 \beta^{*} c^{* 3} k^{*} l^{*}}{\left(2+\beta^{*} c^{* 2} k^{*} / \omega^{* 3}\right)} \frac{c^{*} \overline{v^{*} v^{*}}}{\omega^{* 4}\left(1-c^{* 2} k^{* 2} / \omega^{* 2}\right)^{2}} \\
& =-\left[\frac{2 \delta^{2}}{\left(2+\alpha \delta^{2} \gamma\right)\left(1-\gamma^{2}\right)^{2}} \frac{c^{* 2} k^{*} l^{*}}{\omega^{* 2}}\right] \alpha c^{*} \overline{v^{*} v^{*}},
\end{aligned}
$$

where the last line has been written using the set of nondimensional parameters. These are defined as

$$
\alpha \equiv \beta^{*} c^{*} / f_{0}^{* 2}, \quad \delta \equiv f_{0}^{*} / \omega^{*}, \quad \gamma \equiv c^{*} k^{*} / \omega^{*} .
$$

It can be said that the last line of (24c) represents the contribution of higher order terms in an asymptotic expansion based on $\alpha, \delta$, and $\gamma$. This contribution should not be confused with the universal expression of the additional rotational flux which has already been clarified at (23a) and (23d). It should be also noted that the net content within the square brackets on the last line of (24c) is nondimensional, for which we shall make scale analysis in the next paragraph.

The quantity $\alpha c^{*} \overline{v^{*} v^{*}}$ on the last line of (24c) may be interpreted as a reference for the magnitude of the energy flux of mid-latitude RWs. Mid-latitude RWs may be characterized as

$$
|\alpha \gamma|=\frac{\beta^{*} c^{* 2} / f_{0}^{* 2}}{\left|\omega^{*} / k^{*}\right|} \geq 1, \delta^{2} \gg 1, \quad \gamma^{2} \gg 1 .
$$

Thus, the net content within the square brackets on the last line of (24c) approximates to zero, which justifies (23d) for mid-latitude RWs. On the other hand, for midlatitude IGWs, $c^{*} \overline{v^{*} v^{*}}$ on the last line of (24c) represents a reference for the magnitude of the energy flux. IGWs on an $f$-plane may be characterized as

$$
\alpha=0, \quad \delta^{2} \leq 1, \quad \gamma^{2}<1 .
$$

Thus, the last line of (24c) vanishes, which justifies (23d) for IGWs on an $f$-plane ${ }^{10}$.

To summarize, the streamfunction Eq. (17a) associated with EPV and the universal expression of the additional rotational flux in (17b) applies to both mid-latitude and equatorial waves, in particular for wave types considered in the present study, as listed in Table 2.

\section{Appendix 2}

\section{Approximate expressions for the energy flux}

The exact profile of the group velocity times wave energy is given by the set of (15a) and (16), which is what we call the level-0 energy flux. Owing to the last term on the left hand side of (16) that contains the second-order partial differentiation with respect to time, the procedure of inverting EPV, without using a Fourier analysis, is still complicated.

Hence, we investigate the consequence of artificially removing the second-order time derivative term from (16) as

$$
\nabla^{2} \varphi^{\text {app }}-y^{2} \varphi^{\text {app }}=q,
$$

where the superscript of $\varphi^{\text {app }}$ indicates that the solution of (26a) may be regarded as an approximation for the solution $\varphi$ of the accurate streamfunction Eq. (16) associated with EPV. We have calculated the meridional profiles of

$$
\overline{u p}+\left(\overline{p \varphi^{\mathrm{app}}} / 2+\overline{u_{t t} \varphi^{\mathrm{app}}}\right)_{y},
$$

as shown by the dashed orange lines in Fig. 2 for lowfrequency equatorial waves (e.g., equatorial RWs) and in Fig. 3 for high-frequency equatorial waves (e.g., equatorial IGWs). Since this is an analytical investigation, we have used $\varphi^{\text {app }}=-v_{\theta} /\left(k-\omega^{3}\right)$ which has been derived from the EPV inversion Eq. (26a) with the use of the characteristic Eq. (10). All panels in Fig. 2 show a nice agreement between the dashed orange line given by (26b) and the solid black line, $(\partial \omega / \partial k)\left(\overline{u^{2}+v^{2}+p^{2}}\right)$. By contrast, all panels in Fig. 3 show a finite disagreement between the dashed orange line given by $(26 \mathrm{~b}), \overline{u p}+\left(\overline{p \varphi^{\mathrm{app}}} / 2+\right.$ $\left.\overline{u_{t t} \varphi^{\mathrm{apP}}}\right)_{y}$, and the solid-black line, $(\partial \omega / \partial k)\left(\overline{u^{2}+v^{2}+p^{2}}\right)$.

It would be nice if there is a unified approximation for the energy flux that is able to represent the profile of the group velocity times the energy of both low- and high-frequency equatorial waves. We have found that this requirement is roughly satisfied if (26b) is simplified as

$$
\overline{u p}+\left(\overline{p \varphi^{\text {app }}} / 2\right)_{y},
$$

where $\varphi^{\text {app }}=-v_{\theta} /\left(k-\omega^{3}\right)$ is the solution of (26a). The profile of (26c) is shown by the solid blue lines in Figs. 2 and 3 for low- and high-frequency equatorial waves, respectively. This expression provides what we think is a potentially useful approximation for the group velocity times wave energy (the solid black lines) for all types of equatorial waves, as we show in the "Methods/Experimental" section.

In the present study, (26b) and its vector and dimensional form (18b) are referred to as the level-1 energy flux. Likewise, (26c) and its vector and dimensional form (18c) are referred to as the level-2 energy flux.

Why do we appreciate the level-2 energy flux regardless of the error? An expression for pseudomomentum (or 
wave-activity) flux has long been used for the model diagnosis of the direction of the group velocity of waves in the atmosphere (and also the ocean), including in lowlatitude regions (Ripa 1982; Hoskins et al. 1983; Plumb 1986; Haynes 1988; Randel and Williamson 1990; Brunet and Haynes 1996; Fukutomi and Yasunari 2002; Wakata and Kitaya 2002; Kawatani et al. 2010). Using the analytical solution of equatorial waves, we have calculated the profile of the traditional pseudomomentum flux ${ }^{11}$ times the phase velocity of waves (see Appendix 3), as shown by the purple dots in Figs. 2 and 3. Interestingly, for lowfrequency waves, the profile of the pseudomomentumflux-based expression (the purple dots) is almost the same as that of the level-2 energy flux (the blue solid line). On the other hand, for high-frequency waves, the profile of the pseudomomentum-flux-based expression (the purple dots) is similar to that of the level-1 energy flux (the orange dashed line) and quite different from the exact, level-0 energy flux to which the level-2 energy flux is a better approximation. Thus, the level-2 energy flux is, in general, an improvement on the traditional model diagnosis of group velocity based on the pseudomomentum flux.

Concerning extension to mid-latitude waves, both the level-1 and level-2 energy fluxes satisfy all conditions noted in the last paragraph of the "Boundary conditions and the connection to mid-latitude regions" section. Note that the inversion Eq. (18a) of EPV is seamlessly solvable at all latitudes with the boundary condition of $\varphi^{\text {app } *}=$ 0 . To summarize, the set of (18a) and (18c) [together with the boundary condition (17c)] - what we call the level-2 expression-originates from a trade-off between mathematical exactness and practical accessibility. The mathematical exactness for retrieving the group velocity of equatorial waves times wave energy has been achieved by the set of (17a) and (17b) - what we call the level0 expression. However, its accessibility is harmed by the second-order time derivative term in the streamfunction equation (16) associated with EPV. On the other hand, concerning the practical accessibility, the set of (18a) and (18c) - the level-2 expression-has the advantages that (i) it is seamlessly solvable at all latitudes and (ii) it provides a unified expression for all types of waves with which to estimate the direction of the group velocity. We have noted, for equatorial waves, that the profile of the level-2 energy flux is somewhat better than that of the traditional pseudomomentum flux. It should be also noted that the energy flux given by (18c) satisfies the boundary condition of no flux through coastlines [using (17c)], an issue not considered in previous studies for the pseudomomentum flux. With these requirements in mind, we hope that future studies can lead to either an improved approximation or a numerical algorithm for the level-0 energy flux.

\section{Appendix 3}

Similarity between the level-2 energy flux of this study and the pseudomomentum flux in previous studies

Ripa (1982) has derived a conservation equation for pseudomomentum (or wave activity) associated with ageostrophic waves. His equation may be reproduced using (1a)-(1c) as

$$
\begin{aligned}
& \frac{\partial}{\partial t^{*}} \underbrace{\left(\frac{p^{*} u^{*}}{c^{* 2}}-\frac{q^{* 2}}{2 \beta^{*}}\right)}_{\text {IB pseudomomentum }}+\nabla^{*} \cdot \underbrace{\left\langle\left\langle E^{*}-v^{*} v^{*}, v^{*} u^{*}\right\rangle\right\rangle}_{\mathrm{IB} \text { flux }}=0 \\
& E^{*} \equiv \frac{1}{2}\left(u^{* 2}+v^{* 2}+\frac{p^{* 2}}{c^{* 2}}\right)
\end{aligned}
$$

where the prognostic quantity may be referred to as the impulse-bolus (IB) pseudomomentum (Aiki et al. 2015, hereafter ATG15) and $E^{*}$ is the wave energy. Note that the IB pseudomomentum given here is the shallow water version of that given by Eq. (27a) in ATG15. It has been known that the expression of the flux in (27a) can indicate the direction of the group velocity of different types of waves, in particular, mid-latitude RWs and IGWs (Hoskins et al. 1983; Plumb 1986; Haynes 1988). Another nice feature of the IB pseudomomentum Eq. (27a) is that it does not contain a singularity at the equator. In order to investigate the origin of these features, ATG15 have shown in their Eq. (18a) an identity between the IB pseudomomentum and the classical energy-based (CE) pseudomomentum to read (again, written here for the shallow water equations)

$$
\begin{aligned}
\underbrace{\frac{E^{*}}{\left(\omega^{*} / k^{*}\right)}}_{\text {CE pseudomomentum }}= & \underbrace{\frac{p^{*} u^{*}}{c^{* 2}}-\frac{q^{* 2}}{2 \beta^{*}}}_{\text {IB pseudomomentum }} \\
& -\frac{\partial}{\partial y^{*}}\left(\frac{u^{*} q^{*}}{2 \beta^{*}}\right)+\frac{\partial}{\partial x^{*}}\left(\frac{v^{*} q^{*}}{2 \beta^{*}}\right),
\end{aligned}
$$

which may be derived from (1a)-(1c) of the present study. Application of a low-pass temporal filter to (27b), and then, understanding the phase relationship between $v^{*}=$ $-q_{t^{*}}^{*} / \beta^{*}$ and $q^{*}$ yields

$$
\frac{\overline{E^{*}}}{\left(\omega^{*} / k^{*}\right)}=\frac{\overline{p^{*} u^{*}}}{c^{* 2}}-\frac{q^{* 2}}{2 \beta^{*}}-\frac{\partial}{\partial y^{*}}\left(\overline{\frac{u^{*} q^{*}}{2 \beta^{*}}}\right) .
$$

Substitution of (28b) to a low-pass time-filtered version of (28a) yields

$$
\begin{aligned}
& \frac{\partial}{\partial t^{*}} \overline{E^{*}}+ \\
& \frac{\omega^{*}}{k^{*}} \nabla^{*} \cdot\left\langle\left\langle\overline{E^{*}-v^{*} v^{*}}, \overline{v^{*} u^{*}}+\frac{\partial}{\partial t^{*}}\left(\overline{\frac{u^{*} q^{*}}{2 \beta^{*}}}\right)\right\rangle=0,\right.
\end{aligned}
$$


which is a prognostic equation for the wave energy wherein the zonal component of the flux is proportional to that in the IB pseudomomentum equation (27a).

It is easy to expect that the expression of the flux in (29) can indicate the direction of the group velocity of midlatitude RWs and IGWs (Hoskins et al. 1983; Plumb 1986; Haynes 1988). For equatorial waves, here, we investigate the meridional profile of $\left(\overline{E^{*}-v^{*} v^{*}}\right) \omega^{*} / k^{*}$ as shown by the purple dots in Figs. 2 and 3 for low- and highfrequency waves, respectively. For low-frequency waves (Fig. 2), the meridional profile of $\left(\overline{E^{*}-v^{*} v^{*}}\right) \omega^{*} / k^{*}$ (the purple dots) is almost the same as that of the level-2 energy flux (the blue solid line), showing that the level2 energy flux and the IB flux are closely related. For high-frequency waves (Fig. 3), the meridional profile of $\left(\overline{E^{*}-v^{*} v^{*}}\right) \omega^{*} / k^{*}$ (the purple dots) is nearly the same as that of the level-1 energy flux (the orange dashed line), indicating that the level-2 energy flux is somewhat better than the IB flux.

In fact, without relying on the level-0 expression, we have arrived at the level-2 expression of the energy flux by extending the investigation of ATG15 concerning the algebraic structure of the IB flux (to be explained in a future study). ATG15 have addressed the importance of a waveinduced scalar quantity and symbolized it as $\Lambda$ : it vanishes for mid-latitude IGWs (i.e., waves with no perturbation of EPV) and becomes nonzero for mid-latitude RWs (i.e., wave with a perturbation of EPV). Here, we suggest that $\bar{\Lambda}=\left(\overline{p^{*} \eta^{*}}\right)_{y^{*}} / 2$ is closely linked to $\left(\overline{p^{*} \varphi^{\text {app* }}}\right)_{y^{*}} / 2$ in the present study ( $\eta^{*}$ is meridional displacement). This is why the level-2 expression for the energy flux in the present study can indicate the direction of the group velocity of different types of waves, an issue we shall discuss in a future study.

Note that the IB flux in (27a) has already been used for the model diagnosis of waves in low-latitude regions (Randel and Williamson 1990; Brunet and Haynes 1996; Fukutomi and Yasunari 2002; Wakata and Kitaya 2002; Kawatani et al. 2010). We suggest that, despite the certain inaccuracy associated with equatorial waves as compared with the level-0 expression, the level-2 expression of the energy flux in the present study will be at least as useful as the IB flux which has long been used in the atmospheric (and oceanic) literature. For oceanic applications, the level-2 energy flux brings two new advantages over the IB flux: (i) the level-2 energy flux satisfies a no-normalflux boundary condition at coastlines, and (ii) the wave energy is a sign-definite quantity while the IB pseudomomentum is not.

Overall, we address the balance of (i) model accessibility, (ii) unified treatment for different types of waves, (iii) mathematical accuracy, and (iv) boundary conditions at coastlines. With these requirements in mind, we hope future studies can lead to either an improved approximation or a numerical algorithm for the level-0 energy flux, wherein the profile of the IB flux will provide a reference for accuracy because the IB flux has long been used in previous studies.

\section{Additional file}

Additional file 1: Movie of the model experiment. See the caption of Fig. 4 for details. (MP4 2365 kb)

\section{Abbreviations}

EPV: Ertel's potential vorticity; IGW: Inertia gravity wave; KW: Kelvin wave; RGW: Mixed Rossby-gravity wave; RW: Rossby wave

\section{Acknowledgements}

This manuscript has been improved by comments from two anonymous reviewers. HA thanks Paal Erik Isachsen for the helpful discussions and RJG is grateful to the GEOMAR for ongoing support.

\section{Funding}

This study was supported by JSPS KAKENHI Grant Numbers 26400474 and $15 \mathrm{H} 02129$ and also by the Deutsche Forschungsgemeinschaft as part of the Sonderforschungsbereich 754 "Climate - Biogeochemistry Interactions in the Tropical Ocean," by the German Federal Ministry of Education and Research as part of the cooperative project SACUS (03G0837A), and by the European Union 7th Framework Programme (FP7 2007-2013) under grant agreement 603521 PREFACE project.

\section{Authors' contributions}

HA proposed the topic and performed the analytical investigation. RJG helped write the manuscript. MC helped with the numerical investigation. All authors read and approved the final manuscript.

\section{Competing interests}

The authors declare that they have no competing interest.

\section{Publisher's Note}

Springer Nature remains neutral with regard to jurisdictional claims in published maps and institutional affiliations.

\section{Author details}

${ }^{1}$ Institute for Space-Earth Environmental Research, Nagoya University, Nagoya City, 464-8601 Aichi, Japan. ${ }^{2}$ Application Laboratory, Japan Agency for Marine-Earth Science and Technology, Yokohama, Japan. ${ }^{3}$ GEOMAR Helmholtz-Zentrum für Ozeanforschung Kiel, Kiel, Germany. ${ }^{4}$ Faculty of Mathematics and Natural Sciences, University of Kiel, Kiel, Germany.

Received: 28 June 2016 Accepted: 6 March 2017

Published online: 31 March 2017

\section{References}

Aiki H, Richards KJ (2008) Energetics of the global ocean: the role of layer-thickness form drag. J Phys Oceanogr 38:1845-1869

Aiki H, Takaya K, Greatbatch RJ (2015) A divergence-form wave-induced pressure inherent in the extension of the Eliassen-Palm theory to a three-dimensional framework for waves at all latitudes. J Atmos Sci 72:2822-2849

Anderson DLT, Gill AE (1979) Beta dispersion of inertial waves. J Geophys Res 84:1836-1842

Ascani F, Firing E, McCreary JP, Brandt P, Greatbatch RJ (2015) The deep equatorial ocean circulation in wind-forced numerical solutions. J Phys Oceanogr 45:1709-1734

Brandt P, Funk A, Hormann V, Dengler M, Greatbatch RJ (2011) Interannual atmospheric variability forced by the deep equatorial Atlantic Ocean. Nature 473:497-500

Brandt P, Claus M, Greatbatch RJ, Kopte R, Toole JM, Johns WE (2016) Annual and semi-annual cycle of equatorial Atlantic circulation associated with basin mode resonance. J Phys Oceanogr 46:3011-3029 
Brunet G, Haynes PH (1996) Low-latitude reflection of Rossby wave trains. J Atmos Sci 53:482-496

Cai M, Huang B (2013) A new look at the physics of Rossby waves: a mechanical-Coriolis oscillation. J Atmos Sci 70:303-316

Cane MA, Moore DW (1981) A note on low-frequency equatorial basin modes. J Phys Oceanogr 11:1794-1806

Chelton DB, Schlax MG (1996) Global observations of oceanic Rossby waves. Science 272:234-238

Claus M, Greatbatch RJ, Brandt P (2014) Influence of the barotropic mean flow on the width and the structure of the Atlantic equatorial deep jets. J Phys Oceanogr 44:2485-2497

Claus M, Greatbatch RJ, Brandt P, Toole J (2016) Forcing of the Atlantic equatorial deep jets derived from observations. J Phys Oceanogr 46:3549-3562

Cummins PF, Oey LY (1997) Simulation of barotropic and baroclinic tides off northern British Columbia. J Phys Oceanogr 27:762-781

Fukutomi Y, Yasunari T (2002) Tropical-extratropical interaction associated with the 10-25-day oscillation over the western Pacific during the northern summer. J Meteo Soc Japan 80:311-331

Furuichi N, Hibiya T, Niwa Y (2008) Model-predicted distribution of wind-induced internal wave energy in the world's oceans. J Geophys Res 113:C09034

Gill AE (1982) Atmosphere-ocean dynamics. Academic Press, London

Greatbatch RJ, Brandt P, Claus M, Didwischus S-H, Fu Y (2012) On the width of the equatorial deep jets. J Phys Oceanogr 42:1729-1740

Haynes PH (1988) Forced, dissipative generalizations of finite-amplitude wave-activity conservation relations for zontal and nonzonal basic flows. J Atmos Sci 45:2352-2362

Hoskins BJ, James IN, White GH (1983) The shape, propagation and mean-flow interaction of large-scale weather systems. J Atmos Sci 40:1595-1612

Isachsen PE, LaCasce JJ, Pedlosky J (2007) Rossby wave instability and apparent phase speeds in large ocean basins. J Phys Oceanogr 37:1177-1191

Iwasaki T (2001) Atmospheric energy cycle viewed from wave-mean-flow interaction and Lagrangian mean circulation. J Atmos Sci 58:3036-3052

Johnson GC, Zhang D (2003) Structure of the Atlantic Ocean equatorial deep jets. J Phys Oceanogr 33:600-609

Kawatani Y, Sato K, Dunkerton TJ, Watanabe S, Miyahara S, Takahashi M (2010) The roles of equatorial trapped waves and internal inertia-gravity waves in driving the quasi-biennial oscillation. Part II: three-dimensional distribution of wave forcing. J Atmos Sci 67:981-997

Lübbecke JF, Böning CW, Keenlyside N, Xie S-P (2010) On the connection between Benguela and equatorial Atlantic Ninos and the role of the South Atlantic Anticyclone. J Geophys Res 115:C09015

Longuet-Higgins MS (1964) On group velocity and energy flux in planetary wave motion. Deep-Sea Res 11:35-42

Masuda A (1978) Group velocity and energy transport by Rossby waves. J Oceanogr Soc Jpn 34:1-7

Matsuno T (1966) Quasi-geostrophic motions in the equatorial area. J Meteo Soc Japan 44:25-43

Matthiessen J-D, Greatbatch RJ, Brandt P, Claus M, Didwischus S-H (2015) Influence of the equatorial deep jets on the north equatorial countercurrent. Ocean Dyn 65:1095-1102

McPhaden MJ, Ripa P (1990) Wave-mean flow interactions in the equatorial ocean. Annu Rev Fluid Mech 20:167-205

Merle J (1980) Annual and interannual variability of temperature in the eastern equatorial Atlantic - the hypothesis of an Atlantic El Nino. Oceanol Acta 3:209-220

Nakamura N, Solomon A (2011) Finite-amplitude wave activity and mean flow adjustments in the atmospheric general circulation. Part II: analysis in the isentropic coordinates. J Atmos Sci 68:2783-2799

Niwa Y, Hibiya T (2004) Three-dimensional numerical simulation of M2 internal tides in the East China Sea. J Geophys Res 109:C04027

Orlanski I, Sheldon J (1993) A case of downstream baroclinic development over western north America. Mon Wea Rev 121:2929-2950

Philander SGH (1989) El Nino, La Nina, and the Southern Oscillation. Academic Press, London

Plumb RA (1986) Three-dimensional propagation of transient quasi-geostrophic eddies and its relationship with the eddy forcing of the time mean flow. J Atmos Sci 43:1657-1678
Randel WJ, Williamson DL (1990) A comparison of the climate simulated by the NCAR community climate model (CCM1:R15) with ECMWF analysis. J Climate 3:608-633

Ripa P (1982) Nonlinear wave-wave interactions in a one-layer reduced-gravity model on the equatorial $\beta$ plane. J Phys Oceanogr 12:97-111

Schopf PS, Anderson DLT, Smith R (1981) Beta-dispersion of low-frequency Rossby waves. Dyn Atmos Oceans 5:187-214

Takaya K, Nakamura H (1997) A formulation of a wave activity flux for stationary Rossby waves on a zonally varying basic flow. Geophys Res Lett 24:2985-2988

Thierry V, Treguier AM, Mercier H (2004) Numerical study of the annual and semi-annual fluctuations in the deep equatorial Atlantic Ocean. Ocean Model 6:1-30

Wakata Y, Kitaya S (2002) Annual variability of sea surface height and upper layer thickness in the Pacific Ocean. J Oceanogr 58:439-450

Yanai M, Maruyama T (1966) Stratospheric wave disturbances propagating over the equatorial pacific. J Meteo Soc Japan 44:291-294

Zhai X, Johnson HL, Marshall DP, Wunsch C (2012) On the wind power input to the ocean general circulation. J Phys Oceanogr 42:1357-1365

\section{Submit your manuscript to a SpringerOpen ${ }^{\circ}$ journal and benefit from:}

- Convenient online submission

- Rigorous peer review

- Immediate publication on acceptance

Open access: articles freely available online

- High visibility within the field

- Retaining the copyright to your article

Submit your next manuscript at $>$ springeropen.com 